\title{
Minimum cost path problem for Plug-in Hybrid Electric Vehicles
}

\author{
Okan Arslan, Barış Yıldız, Oya Ekin Karaşan* \\ Bilkent University, Department of Industrial Engineering, Bilkent, 06800 Ankara, Turkey
}

\section{A R T I C L E I N F O}

\section{Article history:}

Received 5 January 2015

Received in revised form 20 March 2015

Accepted 2 May 2015

Available online 11 June 2015

\section{Keywords:}

Dynamic programming

Integer programming

Routing

Long-distance trips

Energy management

\begin{abstract}
A B S T R A C T
We introduce a practically important and theoretically challenging problem: finding the minimum cost path for PHEVs in a road network with refueling and charging stations. We show that this problem is NP-complete and present a mixed integer quadratically constrained formulation, a discrete approximation dynamic programming heuristic, and a shortest path heuristic as solution methodologies. Practical applications of the problem in transportation and logistics, considering specifically the long-distance trips, are discussed in detail. Through extensive computational experiments, significant insights are provided. In addition to the charging infrastructure availability, a driver's stopping tolerance arises as another critical factor affecting the transportation costs.
\end{abstract}

(c) 2015 Elsevier Ltd. All rights reserved.

\section{Introduction}

Electric transportation technology is an emerging challenge for the transportation sector and an opportunity in logistics operations from the environmental and cost perspectives. The interest in electric vehicles (EVs) and their variants such as Plug-in Hybrid Electric Vehicles (PHEVs) is on the rise due to the economic, environmental and security concerns associated with gasoline. A PHEV has an electric motor and an internal combustion engine (ICE) as its power sources. It provides reduction in both transportation costs and greenhouse gas emissions with respect to a comparable conventional vehicle (CV) (Windecker and Ruder, 2013). It has the capabilities of an EV such as charging from a regular power outlet and the convenience of a gasoline powered CV such as long-distance trips. PHEVs operate in either charge depleting (CD) mode or charge sustaining (CS) mode. On CD mode, the electric motor generates the required power and the PHEV uses its batteries as the energy supply. Once a minimum state-of-charge is reached, the PHEV switches to CS mode, and the power that propels the vehicle is mainly generated by the ICE using gasoline as the energy source. Thus, the difference between an EV and a PHEV is the CS mode drive flexibility of the latter (Pistoia, 2010; Axsen and Kurani, 2010; Axsen et al., 2008; Markel and Wipke, 2001; Traut et al., 2011; He et al., 2013). PHEVs can be refueled at regular gasoline stations similar to CVs, or can be charged en-route in charging stations similar to EVs (U.S. Department of Energy, 2008). As such, with its unique capabilities, PHEV technology stands as a major milestone in the road to 'electrification of the automobile' (Tate et al., 2008).

\subsection{Motivation}

In this paper, we introduce the minimum cost path problem for PHEVs (MCPP-PHEV). This problem is fundamental for long-distance PHEV trips that possibly require several refueling/charging stops. As one of the initial steps of

\footnotetext{
* Corresponding author.
} 
renewable energy revolution in transportation, benefits and shortcomings of PHEVs for the urban (short distance) trips are well appreciated and investigated. However, as we review in the related literature below, the long-distance use of PHEVs is not well studied. From the economic and environmental perspectives, long-distance travel is certainly an important subject when the potential benefits of these vehicles are considered, and focusing solely on the short range usage would fail to achieve a complete treatment of the topic. According to the National Household Travel Survey by the U.S. Department of Transportation (2010), the trips by personally owned vehicles that are longer than 50 miles added up to 575 billion miles in 2009 in US. The role of the long-distance trips is even more prominent in the logistics. This article is aimed to be a first attempt to study the long-distance PHEV trips that require several refueling/charging stops.

MCPP-PHEV is a practically important problem for a broad audience. The first and the foremost subjects of interest are the personal PHEV drivers. A recent study by Turrentine and Kurani (2007) reports that personal vehicle drivers lack the basic building blocks of knowledge related to transportation costs. When it comes to PHEVs, the cost components get much more complicated compared to single energy source vehicles, and decision-support models are needed both for the routing and refueling/charging decisions. In this respect, this study offers personal PHEV drivers the possibility of understanding the PHEV economics more clearly. Furthermore, navigation devices might implement the methodologies in this study to offer a valuable decision support tool for the PHEV drivers.

The second major interest group is the logistics firms with a green logistics perspective. Alternative fuel vehicles, and PHEVs in particular, are primarily used by individuals for personal transportation purposes. Nevertheless, a PHEV, with its comparatively long driving-range with respect to an EV, is a promising alternative to be used in logistics. There are different studies directed towards exploring the possible usage of EVs and PHEVs in the future transportation fleets (Juan et al., 2014; Sathaye, 2014; Parish and Pitkanen, 2012; Davis and Figliozzi, 2013). U.S. Department of Energy (2012) also prepared a handbook for fleet managers on the guidelines of incorporating EVs and PHEVs into their fleets. Navigant Research (2013) estimates that fleet purchases of EVs and PHEVs will be more than 291,000 in 2020 worldwide. Production of light, medium and heavy-duty PHEV trucks by several companies (Smith Electric Vehicles, 2015; Odyne Systems, 2015; Business Wire, 2014; Quantum Fuel Systems, 2012; Bloomberg, 2015; Volvo, 2015) is a strong indication of the expected proliferation of PHEVs in logistics fleets highlighting the practical significance of the problem investigated in this paper.

Finally, MCPP-PHEV is also an interesting problem for the governments and infrastructure investors. With economic, security and environmental motivations, governments take an active role in promoting EV and PHEV usage. They provide several incentives and subsidize investments in the infrastructure such as charging stations. Moreover, new incentives started to emerge targeting specifically the business use of PHEVs (United Kingdom Government, 2012; Clean Transport and Technology Limited, 2015; Mitsubishi Cars, 2015). Investigating the long-distance transportation costs of PHEVs, MCPP-PHEV is a significant tool for the governments to better direct their incentive programs and for businesses to assess the opportunities and risks regarding their investment decisions. In summary, MCPP-PHEV is an interesting problem for:

- Personal PHEV drivers who want to learn about the travel costs.

- Firms which want to offer navigation systems tailored for PHEV drivers.

- PHEV truck/van fleets that want to minimize the transportation costs.

- Policy makers from both the auto industries and the governments that decide on the infrastructure establishment incentives.

- Policy makers that decide on the subsidy offers for PHEVs.

\subsection{Related literature}

The use of electric vehicles in the logistics operations led to several new problems flourish in the literature such as pollution-routing problem (Bektaş and Laporte, 2011; Demir et al., 2012, 2014; Franceschetti et al., 2013; Kramer et al., 2015; Koç et al., 2014), green vehicle routing problem (Erdoğan and Miller-Hooks, 2012; Ćirović et al., 2014; Lin et al., 2014; Felipe et al., 2014), location optimization of alternative fuel stations (Kuby and Lim, 2005; Wang and Lin, 2009; Wang and Wang, 2010; Wang and Lin, 2013; Sathaye and Kelley, 2013; Xi et al., 2013; Li and Huang, 2014; Yild1z et al., 2015), and optimal routing problems (Goeke and Schneider, 2015; Schneider et al., 2014; Felipe et al., 2014). The common objective of these studies is to establish the environmental and cost impacts of electric vehicles from the logistics perspective. In this respect, Pelletier et al. (2014) cover possible research perspectives for the goods distribution with electric vehicles including PHEVs.

On the PHEV side, the literature mainly focuses on the energy management problem (Sioshansi, 2012; Flath et al., 2013; Cui et al., 2012) and demand analyses (Golob and Gould, 1998; Dagsvik et al., 2002; Mabit and Fosgerau, 2011; Lopes et al., 2014). In this research, we approach PHEVs from a routing perspective and analyze their travel costs. A driver of a vehicle may prefer to minimize total travel distance, total travel time or total travel cost of a trip, and these problems can basically be modeled as variants of the shortest path problem. In terms of cost, there are various studies that separately investigate the minimum cost path problem for CVs (MCPP-CV) and for EVs (MCPP-EV) as we review below, and polynomial time algorithms 
are proposed for both problems. In this study, we formally present the minimum cost path problem for PHEVs (MCPP-PHEV) and its solution methodologies. To the best of our knowledge, this study is the first attempt to address the MCPP-PHEV.

Several articles address the MCPP-CV in the literature (Ichimori et al., 1981; Lin et al., 2007; Khuller et al., 2007; Lin, 2008a,b; Suzuki, 2008, 2009, 2012; Adler et al., 2013; Jiang and Xie, 2012; Jiang et al., 2012, 2013). Mixed Integer Programming (MIP) formulations, heuristic techniques and linear-time algorithms with dynamic programming approach are proposed as solution methodologies for both fixed and non-fixed path assumptions. Lin (2012) provides interesting complexity results about several variants of MCPP-CV. On the EV side, the problem of energy efficient routing of EVs has been addressed in the literature by considering limited cruising range and regenerative breaking capabilities of EVs (Artmeier et al., 2010; Sachenbacher et al., 2011; Eisner et al., 2011) and polynomial time algorithms have been developed. These problems only consider routing in a network without charging facilities. Kobayashi et al. (2011) and Siddiqi et al. (2011) further include battery charging stations in their models and propose heuristic techniques as solution methodologies. Note that assuming the electricity as a commodity similar to gasoline, the algorithms mentioned above for MCPP-CV can also be used as solution methodologies for MCPP-EV. Routing in a network with battery switching stations is also considered in the literature (Yang and Sun, 2015). Even though it is presented in a different context, Laporte and Pascoal (2011) present a methodology that can also be customized to solve the MCPP-EV problem in a network with battery switching stations. In the existing MCPP-EV studies, battery degradation costs are not considered. Furthermore, all the aforementioned studies consider a single energy source, either gasoline or electricity. Thus, their solution methodologies cannot be directly used for the solution of MCPP-PHEV.

An important problem related to the minimum cost path problems is the shortest weight-constrained path problem (SWCPP) which is known to be NP-complete (Desrosiers et al., 1984; Desrochers and Soumis, 1989). In SWCPP, there are typically two independent measures such as cost and time associated with a path (e.g. Desaulniers and Villeneuve, 2000 and Ahuja et al., 2002). It can efficiently be solved by a shortest path algorithm (i.e. polynomial-time solution algorithms exist) if one of the measures is disregarded or the two measures are consistent. Even though MCPP-PHEV has only the cost measure, we conclude in Section 2 that it is equivalent to SWCPP and thus is NP-complete. Note that MCPP-PHEV is a generalization of MCPP-CV and MCPP-EV.

\subsection{Contributions}

We introduce the MCPP-PHEV and discuss its complexity status and practical uses. The cost components that we consider in this study are the cost of obtaining electricity and gasoline, the battery degradation cost, vehicle depreciation cost and stopping cost. Our study is the first that addresses the battery degradation in the MCPP context. We present a mixed integer quadratically constrained programming (MIQCP) formulation, a discrete approximation dynamic programming heuristic, and a shortest path heuristic as solution methodologies. We also provide significant insights about the effects of driver preferences and the availability of charging stations on the economics of PHEVs.

In the following section, we formally introduce the MCPP-PHEV, prove that it is NP-complete and present an extended version of it. We propose our solution methodologies in Section 3. We present computational results in Section 4 and conclude the study in Section 5 .

\section{Minimum cost path problem for PHEVs (MCPP-PHEV)}

We provide the basic definitions and assumptions necessary for the formalization of MCPP-PHEV. Consider a directed transportation network $G=(N, A)$ and a PHEV traveling from an origin node $s \in N$ to a destination node $t \in N$. Refueling and/or charging stations are located at some of the nodes of the graph and pricing may vary between nodes. Therefore, a PHEV can reduce its travel costs by a proper choice of refueling and/or charging stations.

Proposition 1. If a PHEV does not refuel or charge its battery when traveling from node $i \in N$ to node $j \in N$, then the minimum cost path is the shortest path between nodes $i$ and $j$.

The proof of Proposition 1 is straightforward. Next, we introduce a graph transformation which will be useful for the solution methodologies. A similar construction in a complete different application setting is provided by Chen et al. (2010), Smith et al. (2012) and Yıldız and Karaşan (2015).

Definition 1. Given a weighted graph $G=(N, A)$, let $\hat{N}=\{s, t\} \cup\{i \in N: i$ has a charging and/or refueling station $\}$ and $\hat{A}=\{(i, j): i, j \in \hat{N}$ and $j$ is reachable from $i$ if a PHEV at node $i$ with a full tank of gasoline and fully charged battery can reach node $j$ along a shortest path in $G$. Arc $(i, j) \in \hat{A}$ has a distance equal to the shortest path distance, say $d_{i j}^{\star}$, from $i$ to $j$ in $G$. The graph $\hat{G}=(\hat{N}, \hat{A})$ is called the meta-network of $G$. 
Proposition 1 implies that an optimal solution of a MCPP on a given graph can also be obtained by solving the same MCPP instance on its meta-network. Now, consider nodes $B, C$ and $D$ in graph $G$ in Fig. 1. Only node $C$ has a refueling station. The meta-network $\hat{G}$ is also shown in the same figure. Observe that the arc from $s$ to $t$ is redundant and corresponds to traveling on the path $s \rightarrow C \rightarrow t$. Since the shortest path from $s$ to $t$ contains a node with a refueling station in the original graph $G$, arc $(s, t)$ can be omitted.

Meta-networks can be very dense due to the combined CD and CS mode ranges. The size of the graph is a burden on the solution efficiency, and thus it is useful to omit the redundant arcs in the meta-network. We refer to the graph formed by the omission of redundant arcs as the reduced meta-network denoted by $G^{\prime}$ in Fig. 1. In particular, the arcs that are present in the reduced meta-network $G^{\prime}$ correspond to shortest paths in the original graph $G$ that contain no intermediate nodes with refueling or charging stations.

Definition 2. A vehicle instance (vehicle) is a vector with 6 entries $\langle\bar{P}, \underline{P}, \bar{G}, \underline{G}, \varepsilon, \rho\rangle$ where $\bar{P}$ and $\underline{P}$ are the battery maximum and minimum energy capacities, respectively ( $\mathrm{kW} \mathrm{h}), \bar{G}$ and $\underline{G}$ are the maximum and minimum tank capacities, respectively (gallons), $\varepsilon$ is the average electricity usage ( $\mathrm{kW} \mathrm{h} / \mathrm{mile}$ ) and $\rho$ is the average gasoline usage (gallon/mile).

Definition 3. A network instance (network) is a 7-tuple $\left\langle N, A, s^{e}, s^{g}, c^{e}, c^{g}, d\right\rangle$ where $N, A$ are the sets of nodes and arcs, $s^{e}: N \rightarrow\{0,1\}$ and $s^{g}: N \rightarrow\{0,1\}$ are functions indicating whether a charging or refueling station is located at a node, respectively, $c^{e}: N \rightarrow \mathbb{R}^{+}$is the electricity price function $\left(\mathbb{\leftarrow} / \mathrm{kWh}\right.$ ), $c^{g}: N \rightarrow \mathbb{R}^{+}$is the gasoline price function ( $\left.\mathbb{c} / g a l l o n\right)$ and $d: A \rightarrow \mathbb{R}^{+}$is the length function (miles).

Definition 4. The minimum cost path problem for PHEV (MCPP-PHEV) is defined as finding a path for a vehicle $V$ from a departure node $s$ to a destination node $t$ in a network, and deciding on how much to refuel and where to charge its battery on the path. More formally, the decision version of the problem is:

INSTANCE: $\left\langle V, X, s, t, P_{s}, G_{s}, P_{t}, G_{t}\right\rangle$ where $V$ is a vehicle instance, $X$ is a network instance, nodes $s$ and $t$ are departure and destination nodes, $P_{s}$ and $G_{s}$ are the initial electricity and gasoline storages at node $s, P_{t}$ and $G_{t}$ are the minimum final electricity and gasoline storage requirements at node $t$, respectively, and a positive number $C$.

QUESTION: Is there a path from $s$ to $t$ in network $X$ that can be traveled by vehicle $V$ with initial electricity and gasoline levels of $P_{s}$ and $G_{s}$ and final electricity and gasoline levels of at least $P_{t}$ and $G_{t}$ for a cost less than or equal to $C$ ?

The solution of the MCPP-PHEV is a triplet $\left\langle x, e^{+}, g^{+}\right\rangle$where $x$ is the incidence vector of the optimal path, $e^{+}$and $g^{+}$are vectors of size $|N|$ representing the electricity and gasoline purchases that are transferred to PHEV at each node, respectively.

\subsection{NP-completeness}

Consider the shortest weight-constrained path problem (SWCPP) for directed graphs which is known to be NP-complete (Garey and Johnson, 1979):

INSTANCE: A directed graph $G=(N, A)$ with length $l_{i j} \in \mathbb{Z}^{+}$and weight $w_{i j} \in \mathbb{Z}^{+}$for each $(i, j) \in A$, specified nodes $s, t \in N$ and positive integers $K$ and $W$.

QUESTION: Is there a path in $G$ from $s$ to $t$ with total length $K$ or less and total weight $W$ or less?

First, note that multiplying both $W$ and $w_{i j}, \forall(i, j) \in A$ by a positive constant $\phi$ does not change the solution in SWCPP, and the question in the original instance has a YES answer if and only if the modified instance has a YES answer.

Theorem 1. The MCPP-PHEV is NP-complete.

Proof. Observe that the MCPP-PHEV is in NP: given a solution and a value $C$, one can verify in polynomial time if the solution is feasible and the associated cost is at most $C$. Given an instance $\langle G, l, w, s, t, K, W\rangle$ to SWCPP, let $l^{\min }=\min _{(i, j) \in A} l_{i j}, l^{\max }=$ $\max _{(i, j) \in A} l_{i j}, w^{\max }=\max _{(i, j) \in A} w_{i j}, \phi=\frac{l^{\min }}{2 \times l^{\max } \times w^{\max }}>0, \hat{W}=\phi \times W$ and $\hat{w}_{i j}=\phi \times w_{i j}, \forall(i, j) \in A$. Now, consider an equivalent

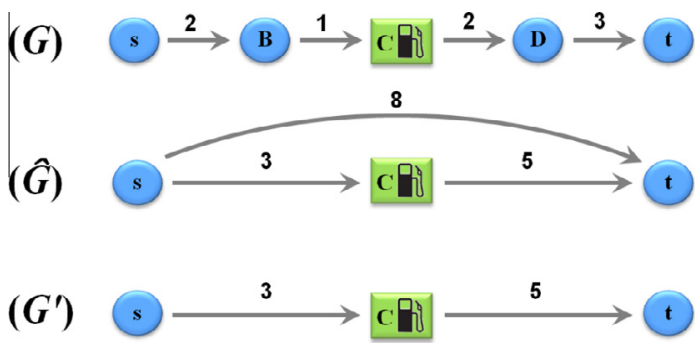

Fig. 1. Graph transformation. 
SWCPP instance $\langle G, l, \hat{w}, s, t, K, \hat{W}\rangle$. We now transform this SWCPP instance into an MCPP-PHEV instance by the following polynomial time transformation: we add a node, say node $i j$, on each $\operatorname{arc}(i, j) \in A$ as shown in Fig. 2 . Let $N^{\prime}$ be the set of newly added nodes, $A_{1}$ be the set of arcs from node $i$ to node $i j, \forall(i, j) \in A$ with distance equal to $\hat{w}_{i j}$ and $A_{2}$ be the set of arcs from node $i j$ to node $j, \forall(i, j) \in A$ with distance equal to 1 mile. The graph is then transformed into $G^{\prime}=\left(N \cup N^{\prime}, A_{1} \cup A_{2}\right)$. In the transformed graph, no gasoline or charging station is located at node $i \in N /\{s\}$. We locate only a refueling station at the source node and the cost of gasoline at this node is $c_{s}^{g}=l^{\max }$. We also locate a charging station, but no refueling station, at every node $i j \in N^{\prime}$ and the cost of electricity at node $i j$ is $c_{i j}^{e}=l_{i j}-\hat{w}_{i j} \times c_{s}^{g}=l_{i j}-\phi \times w_{i j} \times l^{\max }$. Replacing $\phi$, we get $c_{s}^{g}>c_{i j}^{e}>0$ for all nodes $i j \in N^{\prime}$ so that traveling on electricity is always preferable to traveling on gasoline. Let $X$ be this transformed network. Let $V$ be the vehicle $\langle 1,0, \hat{W}, 0,1,1\rangle$. That is, PHEV $V$ has 1 mile of CD mode range and $\hat{W}$ miles of CS mode range. Consider the MCPP-PHEV instance $\langle V, X, s, t, 0,0,0,0\rangle$, i.e. a PHEV $V$ travels from node $s$ to node $t$ in network $X$ with zero initial and final gasoline and electricity levels. Let $K$ be the associated cost input. In Fig. 2, $V$ at node $i$ with minimum electricity level needs to spend $\hat{w}_{i j}$ units of gasoline in order to arrive at node $i j$. Since electricity is preferable to gasoline, it charges its battery fully at node $i j$ and travels to node $j$ on the CD mode. At node $j$, its battery depletes and it starts running on CS mode again. The cost of electricity at node $i j$ and the distance between nodes $i j$ and $j$ are such that the total cost of traversing this arc is $l_{i j}-\hat{w}_{i j} \times c_{s}^{g}$ cents. Observe that the vehicle needs to buy the required level of gasoline at the source node at a cost of $\hat{w}_{i j} \times c_{s}^{g}$ in order to travel from node $i$ to node $j$. Now, it is easy to observe that $V$ has a path from node $s$ to $t$ with cost at most $K$ if and only if the SWCPP has a path from $s$ to $t$ with length at most $K$ and weight at most $\hat{W} \cdot \square$

\subsection{Extensions}

In order to model real world more closely, non-fuel costs such as vehicle depreciation and/or stopping costs need to be taken into account (Suzuki, 2008). To this end, we extend the MCPP-PHEV from three aspects and refer to this problem as the Extended MCPP-PHEV (E-MCPP-PHEV). The first extension is vehicle depreciation cost. A PHEV incurs electricity and gasoline costs while traveling. Furthermore, it loses its value with increasing mileage. Therefore, it incurs a vehicle depreciation cost for every mile traveled. Unless depreciation cost is included in the objective function, an optimal path might get much longer than the shortest path which cannot be tolerated even for the most cost averse driver. Therefore, we indirectly avoid long trip distances by including the depreciation cost in the model. In a sense, the depreciation cost can be considered as the cost of tolerating longer distances, and high depreciation costs would force the E-MCPP-PHEV solutions to follow the shortest path.

Another cost component of a vehicle trip is the stopping cost. This cost component can be a measure of the tolerance for stops on the route. That is, for high enough stopping costs, the optimal solution would be the one with the least number of stops. Note that by including the stopping cost, we avoid excessive number of stops on the optimal path which is not tolerable even for the most cost averse driver.

The battery of a PHEV has a limited lifespan, and its life shortens at each cycle. Thus, apart from the electricity purchase cost, PHEV owners also incur battery degradation cost for each battery charge/discharge cycle. The number of cycles is a nonlinear function of depth of discharge (DoD) as reported by Electric Power Research Institute (2005) and Millner (2010). A sample cycle life function is presented in Fig. 3 by dashed lines. The more the battery is discharged, the less the number of cycles is. For instance, consider a battery worth $\$ 2650$ being discharged to $40 \%$ DoD throughout its lifetime. The expected number of cycles at this DoD is approximately 10000. Therefore each discharging costs the PHEV owner $26.5 \mathbb{\leftarrow}$ (\$2650 $\times 1 / 10000$ ). A sample degradation cost function for a $\$ 2650$ battery is presented in Fig. 3. In our study, we assume that a cycle is completed each time a battery is charged at a station and a PHEV owner incurs a battery degradation cost depending on the DoD level upon arrival to a charging station. We determine this cost by evaluating a quadratic function of DoD. To the best of our knowledge, Sioshansi and Denholm (2010) are the first to include battery degradation cost in their energy management model.

Within this context, the cost components of a PHEV trip are the gasoline cost, the electricity cost, the battery degradation cost, the vehicle depreciation cost and the stopping cost. For simplicity in representing an E-MCPP-PHEV instance, we use the MCPP-PHEV instance representation and assume that all cost components are embedded in the corresponding network instance.

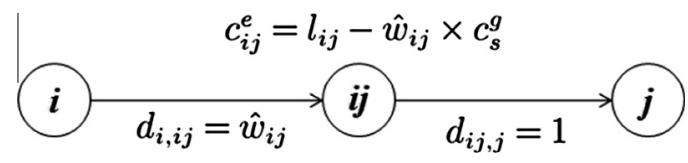

Fig. 2. Graph transformation for Theorem 1. 


\section{Solution techniques}

In this section, we provide a mathematical formulation for the E-MCPP-PHEV. Then we present a dynamic programming approximation heuristic along with its extension and a shortest path heuristic.

\subsection{E-MCPP-PHEV mathematical model}

The parameters and variables to be used in the formulation of the E-MCPP-PHEV are presented below. We assume that the parameters are known with certainty, and the routing decisions are based on the known pricing of electricity and gasoline at every node of the network.
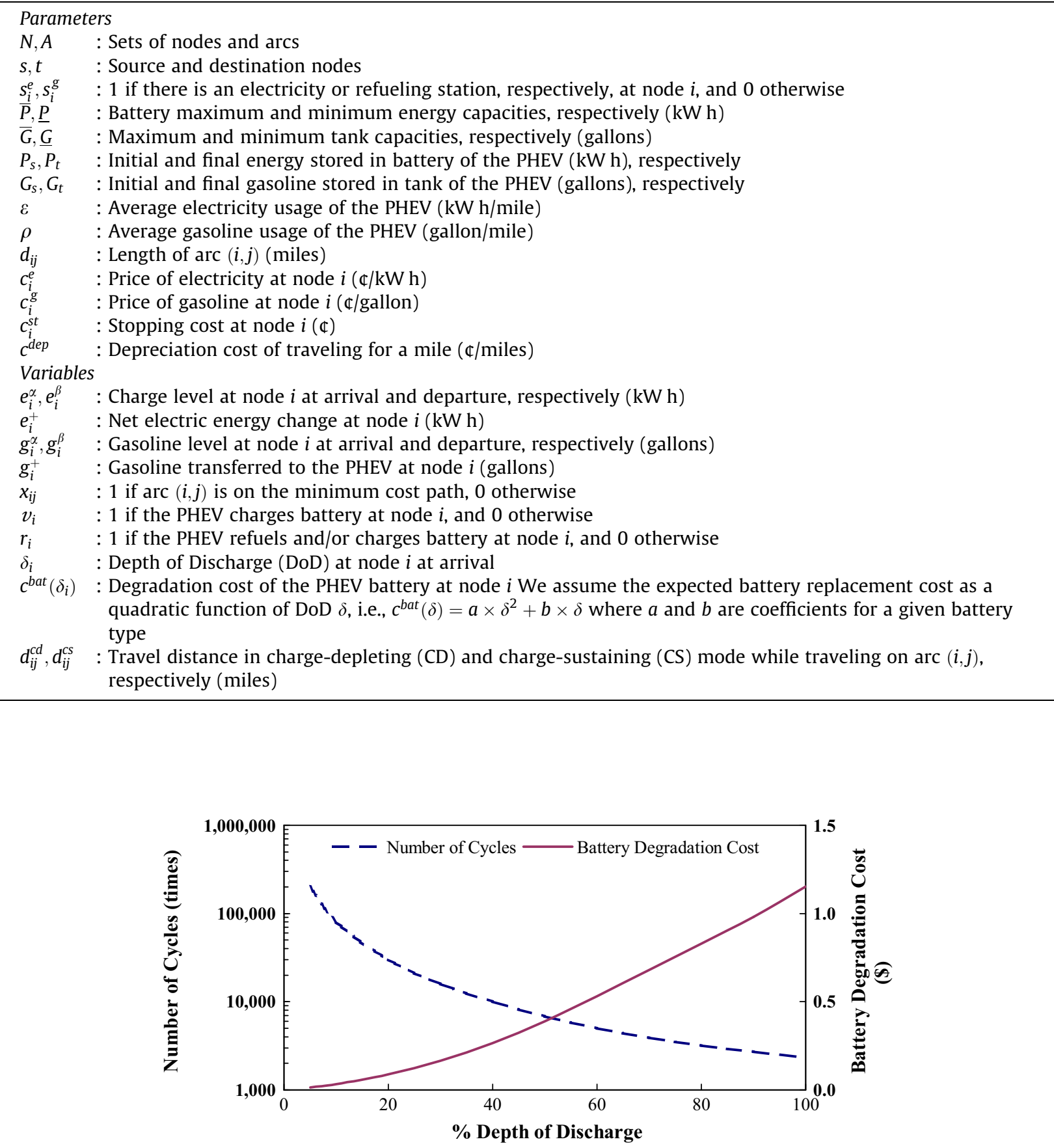

Fig. 3. Cycle life of PHEV batteries as a function of DoD. 
The formulation is as follows:

$$
\text { minimize } \sum_{i \in N} c_{i}^{e} \times e_{i}^{+}+\sum_{i \in N} c_{i}^{g} \times g_{i}^{+}+\sum_{i \in N} c^{b a t}\left(\delta_{i}\right)+\sum_{(i, j) \in A} d_{i j} \times c^{d e p} \times x_{i j}+\sum_{i \in N} c_{i}^{s t} \times r_{i}
$$

subject to

$$
\begin{aligned}
& \sum_{j:(i, j) \in A} x_{i j}-\sum_{j:(j, i) \in A} x_{j i}= \begin{cases}1, & i=s \\
-1, & i=t \\
0, & \forall i \in N /\{s, t\}\end{cases} \\
& e_{i}^{\beta}=e_{i}^{\alpha}+s_{i}^{e} \times e_{i}^{+} \quad \forall i \in N \\
& M \times\left(x_{i j}-1\right) \leqslant e_{j}^{\alpha}-e_{i}^{\beta}+\varepsilon \times d_{i j}^{c d} \leqslant M \times\left(1-x_{i j}\right) \quad \forall(i, j) \in A \\
& \underline{P} \leqslant e_{i}^{\alpha} \leqslant \bar{P} \quad \forall i \in N \\
& \underline{P} \leqslant e_{i}^{\beta} \leqslant \bar{P} \quad \forall i \in N \\
& e_{i}^{+} \leqslant v_{i} \times \bar{P} \quad \forall i \in N \\
& v_{i} \leqslant r_{i} \quad \forall i \in N \\
& e_{s}^{\alpha}=P_{s} \\
& e_{t}^{\alpha} \geqslant P_{t} \\
& \delta_{i}=\frac{e_{i}^{+}}{\bar{P}} \quad \forall i \in N \\
& c^{b a t}\left(\delta_{i}\right) \geqslant a \times\left(\delta_{i}\right)^{2}+b \times \delta_{i}-M \times\left(1-v_{i}\right) \quad \forall i \in N \\
& g_{i}^{\beta}=g_{i}^{\alpha}+s_{i}^{g} \times g_{i}^{+} \quad \forall i \in N \\
& M \times\left(x_{i j}-1\right) \leqslant g_{j}^{\alpha}-g_{i}^{\beta}+\rho \times d_{i j}^{c s} \leqslant M \times\left(1-x_{i j}\right) \quad \forall(i, j) \in A \\
& \underline{G} \leqslant g_{i}^{\alpha} \leqslant \bar{G} \quad \forall i \in N \\
& \underline{G} \leqslant g_{i}^{\beta} \leqslant \bar{G} \quad \forall i \in N \\
& g_{i}^{+} \leqslant r_{i} \times \bar{G} \quad \forall i \in N \\
& g_{s}^{\alpha}=G_{s} \\
& g_{t}^{\alpha} \geqslant G_{t} \\
& d_{i j}^{c s}+d_{i j}^{c d}=d_{i j} \quad \forall(i, j) \in A \\
& x_{i j}, v_{k}, r_{k} \in\{0,1\} ; d_{i j}^{c d}, d_{i j}^{c s}, e_{k}^{\alpha}, e_{k}^{\beta}, e_{k}^{+}, g_{k}^{\alpha}, g_{k}^{\beta}, g_{k}^{+}, \delta_{k}^{\alpha}, c_{k}^{b a t} \geqslant 0 \quad \forall k \in N, \forall(i, j) \in A
\end{aligned}
$$

The objective function minimizes the cost of traveling. The cost components are the cost of obtaining electricity and gasoline, the battery degradation cost, the depreciation cost and the stopping cost. Constraints (2) enforce the solution to be a path from $s$ to $t$. Constraints (3) are the electricity balance equations for nodes. The level of electricity upon leaving node $i$ equals the entering electricity level plus the electricity obtained at node $i$. Similarly, Constraints (4) are the electricity balance equations for those arcs that are on the path. For the non-path arcs, the constraints are relaxed with appropriate big-M values. Constraints (5) and (6) set the upper and lower bounds for the electricity level when entering or leaving a node. Constraints (7) assign binary $v_{i}$ variable a value of 1 if battery is charged at node $i$. Constraints (8) require that $r_{i}$ is set to 1 if $v_{i}$ equals 1 and therefore a stopping cost is incurred in the objective function if the PHEV stops to charge its battery. Constraints (9) and (10) set the electricity level at nodes $s$ and $t$, respectively. Constraints (11) assign proper depth of discharge values and Constraints (12) calculate the battery degradation for each node if battery is charged. Constraints (13)(19) are the counterparts of constraints (3)-(9) for the gasoline case. Constraints (20) ensure that the sum of the distances on CS and CD modes is equal to the arc length if the arc is on the path. Constraints (21) are the domain requirements.

A directed path is an alternating sequence of nodes $\left(n_{0}, n_{1}, n_{2}, \ldots, n_{k}\right)$ with $\left(n_{i}, n_{i+1}\right) \in A, \forall i=0, \ldots, k-1$. A directed path is a non-simple path if it repeats nodes and simple path otherwise. Non-simple paths can occur in transportation networks and as solutions to the E-MCPP-PHEV. The presented MIQCP formulation constructs a simple path in the input network $G=(N, A)$. The input network can be the original transportation network, the meta-network or the reduced meta network, depending on how the non-simple paths are handled. By choosing $G$ as the meta-network or as the reduced meta-network of the input transportation network, a wide group of non-simple paths as potential solutions can be handled by this formulation. All non-simple path occurrences, including extremely rare ones, can be taken into account by duplicating the nodes in $G$ and taking its meta-network at the expense of computational inefficiency. In the Appendix, we present possible occurrences of non-simple paths in the optimal solutions and ways to handle those cases.

Observe that one can easily extract the following information from the outputs of the model: the path to travel from node $s$ to node $t$, how many miles to travel on CD and CS modes on each arc, where to stop to refuel or charge battery, and how much to refuel at each refueling stop. 
The following lemma presents a valid inequality for the E-MCPP-PHEV model.

Lemma 1. $\left(\sum_{i \in N} e_{i}^{+}+P_{s}-P_{t}\right) / \varepsilon+\left(\sum_{i \in N} g_{i}^{+}+G_{s}-G_{t}\right) / \rho \geqslant \sum_{(i, j) \in A} x_{i j} \times d_{i j}$ is a valid inequality to (2)-(21).

The inequality simply states that we need to have enough electricity and gasoline to travel the trip distance. Computational studies in Section 4 show that the above cut is very effective in improving the relaxation bound.

\subsection{Discrete approximation dynamic programming heuristic}

In this subsection, we introduce a dynamic programming based heuristic algorithm referred to as DH. We first define a set of states associated with electricity and gasoline levels at nodes. Then, we present Bellman's equations (Bellman, 1956) that should be satisfied by minimum cost path lengths in order to facilitate the dynamic programming solution methodology. Lastly, we present a graph transformation by which the solution of these equations can be computed efficiently (i.e. in polynomial time) by solving a shortest path problem on the transformed graph.

Definition 5. A state is a triplet $\langle i, \sigma, \lambda\rangle$ which represents the arrival at a node $i \in N$ with $\sigma \in[\underline{P}, \bar{P}] \mathrm{kW}$ h electricity charge and $\lambda \in[\underline{G}, \bar{G}]$ gallons of gasoline. We will use the notation $\omega_{i}^{\sigma, \lambda}$ to refer to a state and replace this notation with $\omega$ or $\omega_{i}$ when the context does not require specific values of $i, \sigma$ and $\lambda$ to be discerned.

Given an E-MCPP-PHEV instance $\left\langle V, X, s, t, P_{s}, G_{s}, P_{t}, G_{t}\right\rangle$, a solution $\left\langle x, e^{+}, g^{+}\right\rangle$contains a path from $s$ to $t$ which can be extracted from the vector $x$. With the specific energy $\left(e^{+}\right)$and gasoline $\left(g^{+}\right)$purchases at the nodes, the distances to be covered in CD and CS modes on this path can easily be extracted. Together with $P_{s}$ and $G_{s}$, the vectors $x, e^{+}$and $g^{+}$induce the levels of state-of-charge and gasoline at arrival to the nodes on the solution path. So, for every solution of the E-MCPP-PHEV, there is a unique sequence of states that represents this solution. Note that in general, the E-MCPP-PHEV has an uncountable number of feasible solutions. Since each of these solutions maps uniquely to a sequence of states, the state space is also uncountable. However, this uncountable state space can be approximated with a finite one which is the main idea behind the $\mathrm{DH}$.

Let $\xi, \tau \in \mathbb{N}$ be the discretization parameters for the state space. Consider two sets $\Sigma=\left\{\sigma_{0}, \sigma_{1}, \ldots, \sigma_{\xi}\right\}$ and $\Lambda=\left\{\lambda_{0}, \lambda_{1}, \ldots, \lambda_{\tau}\right\}$ where $\sigma_{0}=\underline{P}, \lambda_{0}=\underline{G}, \sigma_{k}=\sigma_{0}+k \times \frac{\bar{P}-\underline{P}}{\underline{\xi}} \quad \forall k \in\{1,2, \ldots, \xi\}$ and $\lambda_{l}=\lambda_{0}+l \times \frac{\bar{G}-\underline{G}}{\tau} \quad \forall l \in\{1,2, \ldots, \tau\}$. Every $\sigma_{i}$ represents the interval of electricity levels $\left[\sigma_{i}, \sigma_{i+1}\right] \quad \forall i \in\{0,1, \ldots, \xi-1\}$ and $\sigma_{\xi}$ represents the fully charged battery. The representation for each $\lambda$ is similar.

For a given E-MCPP-PHEV instance $\left\langle V, X, s, t, P_{s}, G_{s}, P_{t}, G_{t}\right\rangle, \xi$ and $\tau$ values, we define the discrete state space $\Omega$ as:

$$
\Omega=\left\{\left(\omega_{i}^{\sigma, \lambda} \mid i \in N-\{s, t\}, \sigma \in \Sigma, \lambda \in \Lambda\right\} \cup\left\{\omega_{s}^{P_{s}, G_{s}}, \omega_{t}^{P_{t}, G_{t}}\right\}\right.
$$

Observe that the cardinality of the discrete state space $\Omega$ is bounded by $n \times(\xi+1) \times(\tau+1)$ where $n$ is the number of nodes in $X$, and is finite. Algorithm DH uses $\Omega$ and incurs an approximation error on representing the amount of electricity charge and gasoline left with the PHEV arriving at a node. Obviously this approximation error can be reduced arbitrarily by choosing $\xi$ and $\tau$ large enough.

Definition 6. $\pi: \Omega \rightarrow \mathbb{R}$ is called the value function and $\pi\left(\omega_{i}^{\sigma, \lambda}\right)$ is defined to be the optimal solution value of the E-MCPP-PHEV instance $\left\langle V, X, s, i, P_{s}, G_{s}, \sigma, \lambda\right\rangle$.

The minimum cost transition function $f: \Omega \times \Omega \rightarrow \mathbb{R}^{+}$takes two states $\omega_{i}^{\sigma, \lambda}, \omega_{j}^{\bar{\sigma}, \bar{\lambda}}$ as its arguments and returns the minimum cost of the transition from node $i$ starting with $\sigma \mathrm{kW}$ h charge and $\lambda$ gallons of gasoline to node $j$ ending with at least $\bar{\sigma}$ $\mathrm{kW}$ h charge and $\bar{\lambda}$ gallons of gasoline. When calculating $f\left(\omega_{i}^{\sigma, \lambda}, \omega_{j}^{\bar{\sigma}, \bar{\lambda}}\right)$, we only consider how much to refuel and charge at node $i$. Four cases as detailed below should be considered. A feasibility condition is stated for each case. The cost value is as presented if the feasibility condition is met, and is not finite otherwise. Let $d^{\star}$ represent the shortest path lengths.

- Case 1: No battery charging and no refueling.

Feasibility Condition: The existing electricity charge and gasoline are enough to travel from node $i$ to node $j$ while satisfying the end-state conditions, i.e.,

$$
\sigma \geqslant \bar{\sigma}, \lambda \geqslant \bar{\lambda} \text { and } \frac{(\sigma-\bar{\sigma})}{\varepsilon}+\frac{(\lambda-\bar{\lambda})}{\rho} \geqslant d_{i j}^{\star}
$$

Total Cost: The only cost component to be incurred is the depreciation cost. Thus, $f_{1}\left(\omega_{i}^{\sigma, \lambda}, \omega_{j}^{\bar{\sigma}, \bar{\lambda}}\right)=c^{d e p} \times d_{i j}^{\star}$.

- Case 2: Refueling but no battery charging.

Feasibility Condition: The existing electricity charge and full tank of gasoline are enough to travel from node $i$ to node $j$ while satisfying the end-state conditions, i.e.,

$$
s_{i}^{g}=1, \sigma \geqslant \bar{\sigma} \text { and } \frac{(\sigma-\bar{\sigma})}{\varepsilon}+\frac{(\bar{G}-\bar{\lambda})}{\rho} \geqslant d_{i j}^{\star} .
$$


Total Cost: The minimum cost transition requires to use $(\sigma-\bar{\sigma})$ electricity charge first. Thus $d_{i j}^{c d}=\min \left\{d_{i j}^{\star}, \frac{(\sigma-\bar{\sigma})}{\varepsilon}\right\}$ and $d_{i j}^{c s}=d_{i j}^{\star}-d_{i j}^{c d}$. On the other hand, we need to purchase enough gasoline at node $i$ to cover the travel distance and retain $\bar{\lambda}$ gallons of gasoline at node $j$, i.e., $g_{i}^{+}=\left(d_{i j}^{c s} \times \rho+\bar{\lambda}-\lambda\right)^{+}$gallons of gasoline should be purchased at node $i$. Note that, by the feasibility condition, we make sure that the purchased gasoline is between the limits, i.e. $0 \leqslant g_{i}^{+} \leqslant \bar{G}-\lambda$. Since the battery is not charged, only the gasoline cost, vehicle depreciation cost and stopping cost are included in the total cost function which is $f_{2}\left(\omega_{i}^{\sigma, \lambda}, \omega_{j}^{\bar{\sigma}, \bar{\lambda}}\right)=c_{i}^{g} \times g_{i}^{+}+c^{d e p} \times d_{i j}^{\star}+c_{i}^{s t}$.

- Case 3: Battery charging but no refueling.

Feasibility Condition: A full battery charge and existing level of gasoline are jointly enough to travel from node $i$ to node $j$ while satisfying the end-state conditions, i.e.,

$$
s_{i}^{e}=1, \lambda \geqslant \bar{\lambda} \text { and } \frac{(\bar{P}-\bar{\sigma})}{\varepsilon}+\frac{(\lambda-\bar{\lambda})}{\rho} \geqslant d_{i j}^{\star} .
$$

Total Cost: With the analogous arguments presented for the previous case, we have $e_{i}^{+}=\left(d_{i j}^{c d} \times \varepsilon+\bar{\sigma}-\sigma\right)^{+}$, where $d_{i j}^{c d}=d_{i j}^{\star}-d_{i j}^{c s}$ and $d_{i j}^{c s}=\min \left\{d_{i j}^{\star}, \frac{(\lambda-\bar{\lambda})}{\rho}\right\}$. We do not purchase gasoline in this case. The electricity cost, battery degradation cost, vehicle depreciation cost and stopping cost are included in the total cost. Thus the total cost is, $f_{3}\left(\omega_{i}^{\sigma, \lambda}, \omega_{j}^{\bar{\sigma}, \bar{\lambda}}\right)=c_{i}^{e} \times e_{i}^{+}+c^{b a t}\left(\frac{\bar{P}-\sigma}{\bar{P}}\right)+c^{d e p} \times d_{i j}^{\star}+c_{i}^{s t}$.

- Case 4: Both battery charging and refueling.

Feasibility Condition: A full battery charge and a full tank of gasoline are enough to travel from node $i$ to node $j$, while satisfying the end-state conditions, i.e.,

$$
s_{i}^{e}=1, s_{i}^{g}=1 \text { and } \frac{(\bar{P}-\bar{\sigma})}{\varepsilon}+\frac{(\bar{G}-\bar{\lambda})}{\rho} \geqslant d_{i j}^{\star} .
$$

Total Cost: We define the excess-distance $\bar{d}_{i j}=\left(d_{i j}^{\star}-\frac{(\sigma-\bar{\sigma})^{+}}{\varepsilon}-\frac{(\lambda-\bar{\lambda})^{+}}{\rho}\right)$ as the additional mileage that cannot be covered by the existing charge and fuel levels before charging or refueling at node $i$. Observe that depending on the cost of electricity and gasoline at node $i$, it is cheaper to drive on either CD or CS mode to cover $\bar{d}_{i j}$.

- If CD mode is cheaper, i.e. $\left(c_{i}^{e} \times \varepsilon\right)<\left(c_{i}^{g} \times \rho\right)$, we check if a fully charged battery is enough to cover the required distance $\bar{d}_{i j}$, i.e. $\left(d_{i j}^{c d}+\bar{d}_{i j}\right)<\frac{\bar{P}-\bar{\sigma}}{\varepsilon}$. If so, we have $e_{i}^{+}=\bar{d}_{i j} \times \varepsilon+(\bar{\sigma}-\sigma)^{+}$and $g_{i}^{+}=(\bar{\lambda}-\lambda)^{+}$. Otherwise, $e_{i}^{+}=(\bar{P}-\sigma)$ and $g_{i}^{+}=\left(\left(d_{i j}^{\star}-\frac{\bar{P}-\bar{\sigma}}{\varepsilon}\right) \times \rho+\bar{\lambda}-\lambda\right)^{+}$.

- If CS mode is cheaper, all the calculations to find $e_{i}^{+}$and $g_{i}^{+}$values are just symmetric to the ones presented above and here we skip the details for the sake of brevity.

Once we find the electricity and gasoline amounts $e_{i}^{+}, g_{i}^{+}$, we can easily calculate the total cost $f_{4}\left(\omega_{i}^{\sigma, \lambda}, \omega_{j}^{\bar{\sigma}, \bar{\lambda}}\right)=c_{i}^{e} \times e_{i}^{+}+c_{i}^{g} \times g_{i}^{+}+c^{b a t}\left(\frac{\bar{P}-\sigma}{\bar{P}}\right)+c^{d e p} \times d_{i j}^{\star}+c_{i}^{s t}$.

Considering all four cases, the minimum cost transition function is defined as:

$$
f(\omega, \bar{\omega})=\min _{i \in\{1,2,3,4\}}\left\{f_{i}(\omega, \bar{\omega})\right\}
$$

The following Bellman's equations are based on the principle of optimality:

$$
\begin{aligned}
& \pi\left(\omega_{s}^{P_{s}, G_{s}}\right)=0 \\
& \pi(\omega)=\min _{\bar{\omega} \in \Omega}\{\pi(\bar{\omega})+f(\bar{\omega}, \omega)\} \quad \forall \omega \in \Omega
\end{aligned}
$$

Definition 7. $\tilde{G}=(\Omega, \tilde{A})$ is called the $D H$-Graph where the node set is the discrete state space $\Omega$. The arc set $\tilde{A}$ includes an arc between states $\omega_{i}$ and $\omega_{j} \in \Omega$ with a cost of $f\left(\omega_{i}, \omega_{j}\right)$ if this cost is finite.

Once the DH-Graph is obtained, solving the Bellman's equations, which is the core of the DH algorithm, reduces to solving the shortest path problem on $\tilde{G}$ from state $\omega_{s}^{P_{s}, G_{s}}$ to the state $\omega_{t}^{P_{t}, G_{t}}$. Observe that arcs on the shortest path contain the information where the PHEV stops for refueling/charging and how much electricity charge/gasoline to purchase at those stops. So obtaining the shortest path in $\tilde{G}$ is sufficient to obtain a solution for the E-MCPP-PHEV instance.

$\tilde{G}$ contains $|\Omega|$ nodes and the cardinality of the arc set $\tilde{A}$ is bounded by $|\Omega|^{2}$. Constant time calculation of the transition function $f$ results in $O\left(|\Omega|^{2}\right)$ run time bound for the generation of the DH-Graph. Using Dijkstra's algorithm to find the shortest path in $\tilde{G}$, the overall run time complexity of DH becomes $O\left(|\Omega|^{2}\right)$. 


\subsection{Enhancing the DH Heuristic (DHE)}

Due to the discrete approximation of the true levels of gasoline and electricity, $\mathrm{DH}$ might not always give the optimal solution in terms of refueling and battery charging amounts even if the optimal path is correctly identified. To that end, we provide the enhanced version of DH (DHE) in which we take into account the path that is given by the algorithm, but not the refueling and battery charging decisions. Instead, we consider the subgraph that consists of only the path nodes and the path arcs. Then, we solve the model presented in Section 3.1 on this subgraph. Since the subgraph size is much smaller than the original graph, the solution times of the model formulation reduce drastically and we attain improved refueling and battery charging strategies.

\subsection{Shortest Path Heuristic (SP)}

Minimizing the operating cost on the shortest path is a commonly used solution technique to solve the minimum cost path problems in the literature. Since well known polynomial-time algorithms are available for finding shortest paths, such heuristics are also pervasive in industrial and commercial applications as well. In this context, we propose to solve MIQCP model considering the shortest path as the input graph and optimizing only the refueling and charging decisions.

\section{Computational study}

To test the performances of the proposed solution methodologies and drive insights about the solutions, we conducted extensive numerical experiments using problem instances that represent various network structures and user behaviors. The experiments are carried out on a workstation with $2.66 \mathrm{GHz}$ Xeon processor and $8 \mathrm{~GB}$ memory. We present the data and the results related to computational performances and several measures in the following subsections. It is important to note that with several preliminary experimentations, we have observed that working with reduced meta-networks is satisfactory in capturing the non-simple paths that might arise in our instances and opted to using reduced meta-networks throughout our computational experiments.

\subsection{Data}

A 2013 Chevrolet Volt PHEV has the following specifications: $16.5 \mathrm{~kW} \mathrm{~h}$ battery capacity, 9.3 gallon tank capacity, $0.352 \mathrm{~kW}$ h per mile and 0.027 gallons per mile (U.S. Department of Energy, 2013) usages. In order to avoid over-charging degradation, we assume $20 \%$ and $85 \%$ as the minimum and maximum battery charge levels respectively. Hence, the simulated vehicle has a hard bound of $14 \mathrm{~kW}$ h on capacity rather than $16.5 \mathrm{~kW} \mathrm{~h}$. The battery cost of PHEV is assumed to be $\$ 2650$ and the cost function with respect to depth of discharge is $c^{d e p}(\delta)=79.517 \times \delta^{2}+37.854 \times \delta$, as presented in Fig. 3. We also assume that the minimum tank capacity is zero and the depreciation cost is $1 \overleftarrow{\leftarrow / m i l e . ~ F i v e ~ d i f f e r e n t ~ s t o p p i n g ~}$ cost values are tested: $0,50,100,200$ and $500 \uparrow$.

For the network instances, we consider square mesh shaped networks of node sizes $6 \times 6,7 \times 7,8 \times 8,9 \times 9$ and $10 \times 10$. We generate 10 instances of each size. Every node in a given network is connected with an arc to the next node on the right, left, top and bottom, if there is one. The source and destination nodes are the top left and bottom right nodes of the graph, respectively. The arc distances are random values uniformly distributed between 20 and 40 miles. This is to simulate the fact that real road networks distances between two points could be quite different from Euclidean distances in a plane due to elevation differences, geographical obstacles etc. A refueling station is located at every node and the gasoline prices are uniformly generated in $\$ 3.5$ and $\$ 4.1$ range. We assume that battery charging stations are located randomly at 0\%, 25\%, 50\%, $75 \%$ and $100 \%$ of the total nodes and the electricity prices at charging stations change uniformly between $10 \leftarrow$ and $12 \lessdot$. In total, we have 250 mesh shaped networks and 5 different stopping cost values, i.e. 1250 runs. For each set of parameters, we report the averages corresponding to 10 network instances.

It is clear that mesh networks cannot fully capture all the aspects of a real network. Our main motivation in using such networks is to be able to have an extensive problem set to conduct our numerical experiments. As a better approximation of the real world problem instances, we also consider California road network which will be elaborated in Section 4.4 .

\subsection{Performances of the solution techniques}

We present the basic computational performance measures of the solution methodologies in Table 1 . DH is solved with two different levels of approximation $\xi=\tau=4$ and $\xi=\tau=1$, which we refer to as DH4 and DH1, respectively. The percentage of the optimal solutions for DH4 (DH1) range in 46.8-54.0\% (46.4-54.0\%) for all instances, which is improved by the extended versions of the algorithms to around 89.6-96.8\% (83.2-92.8\%). An optimal path is found by DH4 (DH1) in around 84.4-94.8\% (77.2-91.2\%) of all the instances. Since a high percentage of the optimal solutions (ranging between 64.8-84.8\%) coincide with the shortest paths, the SP heuristic also performs well in minimum cost path problems. DHE1 and SP have very similar run times and both can solve very large problem instances quite fast. However, emphasizing the merits of the 
Table 1

Computational results.

\begin{tabular}{|c|c|c|c|c|c|c|c|}
\hline Node number & $\begin{array}{l}\text { Solution } \\
\text { technique }\end{array}$ & $\begin{array}{l}\text { Opt. sol. } \\
\text { found (\%) }\end{array}$ & $\begin{array}{l}\text { Avg opt. } \\
\text { gap (\%) }\end{array}$ & $\begin{array}{l}\text { Opt. path } \\
\text { found (\%) }\end{array}$ & $\begin{array}{l}\text { Is shortest } \\
\text { path? (\%) }\end{array}$ & $\begin{array}{l}\text { Avg deviation from the } \\
\text { shortest path (\%) }\end{array}$ & CPU seconds \\
\hline \multirow[t]{6}{*}{6} & MIQCP & 100.0 & 0.000 & 100.0 & 79.2 & 0.364 & 1.828 \\
\hline & $\mathrm{DH} 4$ & 54.0 & 0.688 & 93.2 & 80.8 & 1.212 & 0.585 \\
\hline & DH1 & 54.0 & 1.410 & 90.4 & 78.8 & 2.344 & 0.004 \\
\hline & DHE4 & 95.2 & 0.009 & 93.2 & 80.8 & 1.212 & 1.532 \\
\hline & DHE1 & 92.0 & 0.031 & 90.4 & 78.8 & 2.344 & 0.928 \\
\hline & SP & 79.2 & 0.424 & 79.2 & 100.0 & 0.000 & 0.858 \\
\hline \multirow[t]{6}{*}{7} & MIQCP & 100.0 & 0.000 & 100.0 & 84.8 & 0.382 & 2.947 \\
\hline & $\mathrm{DH} 4$ & 52.0 & 0.685 & 94.8 & 82.4 & 1.409 & 1.277 \\
\hline & DH1 & 52.0 & 1.499 & 91.2 & 81.2 & 3.030 & 0.005 \\
\hline & DHE4 & 96.8 & 0.004 & 94.8 & 82.4 & 1.409 & 2.327 \\
\hline & DHE1 & 92.8 & 0.044 & 91.2 & 81.2 & 3.030 & 1.060 \\
\hline & $\mathrm{SP}$ & 84.8 & 0.399 & 84.8 & 100.0 & 0.000 & 1.002 \\
\hline \multirow[t]{6}{*}{8} & MIQCP & 100.0 & 0.000 & 100.0 & 69.6 & 0.350 & 8.696 \\
\hline & DH4 & 49.6 & 0.731 & 89.6 & 67.6 & 1.664 & 2.418 \\
\hline & DH1 & 49.6 & 1.539 & 84.0 & 64.0 & 5.044 & 0.010 \\
\hline & DHE4 & 92.8 & 0.027 & 89.6 & 67.6 & 1.664 & 3.581 \\
\hline & DHE1 & 86.8 & 0.058 & 84.0 & 64.0 & 5.044 & 1.173 \\
\hline & $\mathrm{SP}$ & 69.6 & 0.562 & 69.6 & 100.0 & 0.000 & 1.030 \\
\hline \multirow[t]{6}{*}{9} & MIQCP & 100.0 & 0.000 & 100.0 & 75.2 & 0.337 & 33.105 \\
\hline & DH4 & 50.8 & 0.726 & 88.8 & 74.4 & 1.542 & 4.281 \\
\hline & DH1 & 50.4 & 1.627 & 82.8 & 70.8 & 3.593 & 0.017 \\
\hline & DHE4 & 91.6 & 0.030 & 88.8 & 74.4 & 1.542 & 5.579 \\
\hline & DHE1 & 87.2 & 0.069 & 82.8 & 70.8 & 3.593 & 1.325 \\
\hline & SP & 75.2 & 0.404 & 75.2 & 100.0 & 0.000 & 1.196 \\
\hline \multirow[t]{6}{*}{10} & MIQCP & 100.0 & 0.000 & 100.0 & 64.8 & 0.473 & 184.848 \\
\hline & $\mathrm{DH} 4$ & 46.8 & 0.766 & 84.4 & 62.4 & 1.992 & 6.916 \\
\hline & DH1 & 46.4 & 1.683 & 77.2 & 58.0 & 5.554 & 0.028 \\
\hline & DHE4 & 89.6 & 0.023 & 84.4 & 62.4 & 1.992 & 8.284 \\
\hline & DHE1 & 83.2 & 0.058 & 77.2 & 58.0 & 5.554 & 1.359 \\
\hline & SP & 64.8 & 0.646 & 64.8 & 100.0 & 0.000 & 1.231 \\
\hline
\end{tabular}

proposed dynamic programming approximation, DHE1 performs at least as good as SP for the problem instances considered in this study.

We observe that the solution times for the MIQCP start getting prohibitive as the node number increases. Beyond 100 nodes, there exist problem instances with more than 60 -min solution times. On the other hand, observe that the average solution time of the DH1 is less than $0.028 \mathrm{~s}$ on all network sizes. In fact, the average runtime of DH1 for problem instances with 900 nodes is only $40.3 \mathrm{~s}$ which makes it the suitable solution technique for devices with limited computational capacity. However, since other solution techniques did not scale up to such dimensions, these results are not presented here.

One important fact to note is that the valid inequality presented in Section 3.1 greatly contributes to the solution times of the MIQCP. The average gap of the LP relaxation solution from the optimal solution with and without the cut is $24.89 \%$ and $90.02 \%$, respectively. We also observe that optimal paths of DH4 (DH1) coincide with the shortest paths on the average 62.4-82.4\% (58.0-81.2\%) of the instances. On the average, the deviation from the shortest path changes in the range of $1.21-1.99 \%$ (2.34-5.55\%) of the shortest path distance.

\subsection{Insights}

The cost reduction of a PHEV trip with respect to a CV is mainly due to the CD mode driving technology. How much benefit can be attained is related to the $C D$ mode driving mileage which is dependent on the number of charging stations in the network and the driver's tolerance for stopping. In our numerical experiments, we investigate the effects of these two main parameters: the percentage of nodes with charging stations (which we refer to as the penetration level) and the stopping costs (higher stopping costs imply less tolerance for stopping). In the following graphs, we present the results for the optimal solutions obtained by the MIQCP formulation for 100 nodes network instances. The results for 36, 49, 64 and 81 nodes network instances follow very similar trends to those that we present in these graphs and hence are not presented.

Fig. 4 depicts the average miles per stop for different stopping costs. In order to depict the sole effect of the stopping cost on the average miles per stop, $100 \%$ penetration is chosen. In other words, a PHEV can stop at every node in the network in order to refuel or charge its battery. Observe that lower stopping costs result in frequent stops. This graph can be used for quantifying one's own stopping cost. Knowing the tolerance for average miles between stops, one can easily obtain his/her 


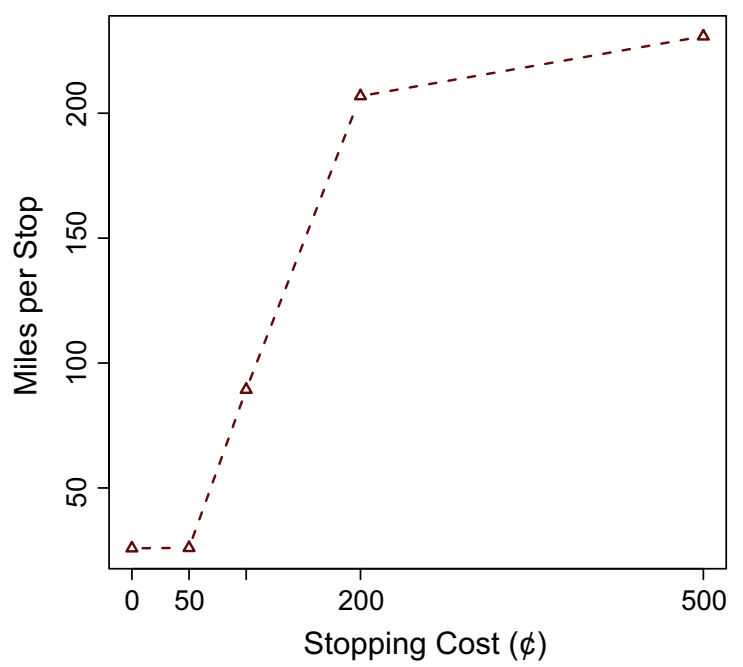

Fig. 4. Average miles per stop for different stopping costs in a network with 100 nodes and $100 \%$ charging station penetration level.

dollar value for stopping cost. On the other hand, the graph can also be used to determine how many stops one can tolerate in a trip and the opportunity cost associated with the time spent in these stops.

Fig. 5 shows the percentage of the distance covered in $C D$ mode. At zero penetration level, there does not exist any charging station in the network and the CD mode mileage is therefore zero. With increasing penetration level, the CD mode mileage increases accordingly. For zero stopping cost, the CD mode trip percentage increases to almost $100 \%$ for $100 \%$ penetration level. On the other hand, for the stopping costs of more than $200 \mathbb{\$}$, the CD mode trip percentage does not go above $15 \%$. This is due to the fact that even though there exists charging opportunities on the path, the driver cannot tolerate for frequent stops and therefore continues on the CS mode rather than CD mode. This implies that for those drivers with less tolerance for stopping, increasing the number of charging stations does not necessarily imply more CD mode drive. Increasing the battery capacity is more important. On the other hand, if the drivers are more tolerant for stopping, increasing the number of charging stations is equivalent to increasing the battery capacity in terms of CD mode drive percentage. Observe that this result is crucial for both infrastructure investors and governments. We believe that decision makers need to consider the drivers' tolerance for stopping which is neglected in the existing literature and more research must be directed towards determining the utility functions of PHEV drivers' willingness for making frequent stops.

The cost per mile graph is depicted in Fig. 6 for different stopping costs and penetration levels. When solving the MIQCP model, the objective function includes the stopping cost, but the cost in the graph is composed of only the following components: electricity cost, gasoline cost, depreciation cost and battery degradation cost. This way, we are able to compare the

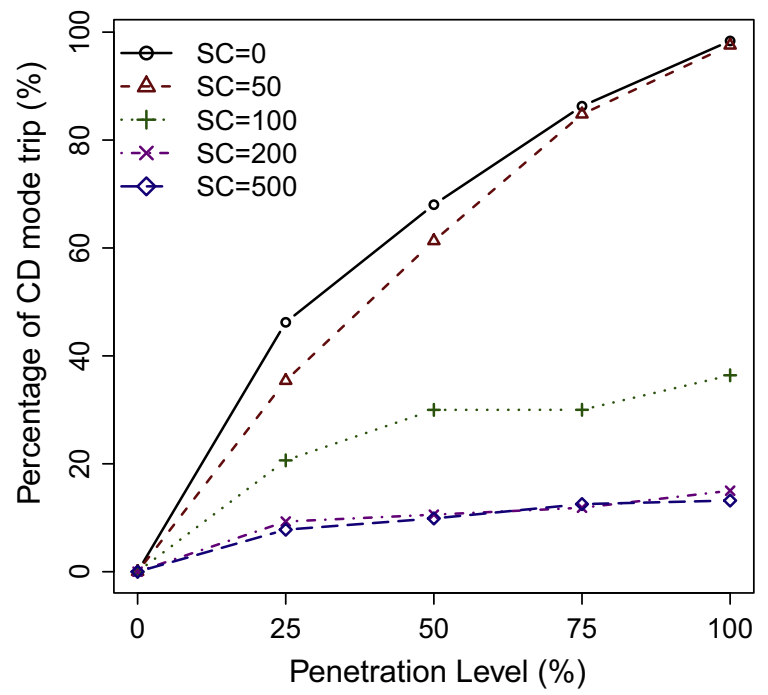

Fig. 5. CD mode trip percentage change for different Stopping Costs (SC) and penetration levels. 
costs for different stopping cost configurations. Observe that Fig. 6 proposes similar results to previous findings. Consider zero stopping cost. As the penetration level increases, the cost per mile decreases to $7 \mathbb{\leftarrow}$ for $100 \%$ penetration level. This result is due to more CD mode trip which can also be observed in Fig. 5. The decrease is not as high for $100 ₫$ stopping cost case. Note that the cost is almost not affected by penetration level increase for higher stopping costs. These results are also parallel to those in Fig. 5.

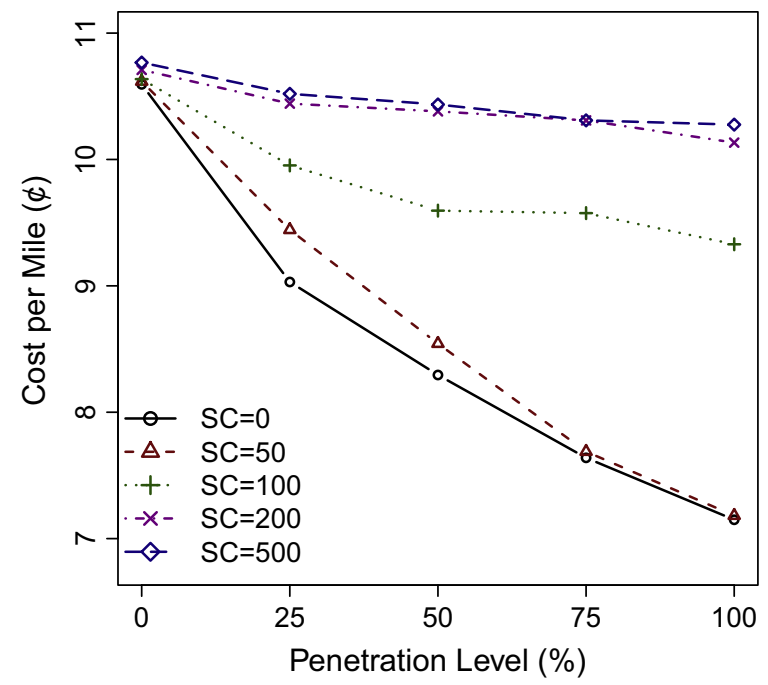

Fig. 6. The effect of charging station penetration level on the cost per mile for different Stopping Costs (SC).

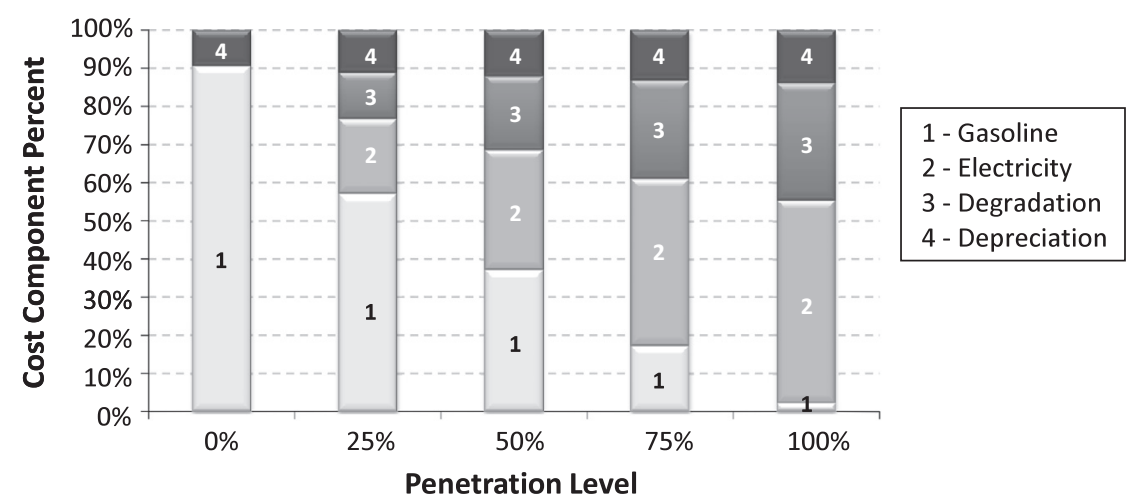

Fig. 7. The effect of charging station penetration level on the cost components for $0 \llbracket$ stopping cost.

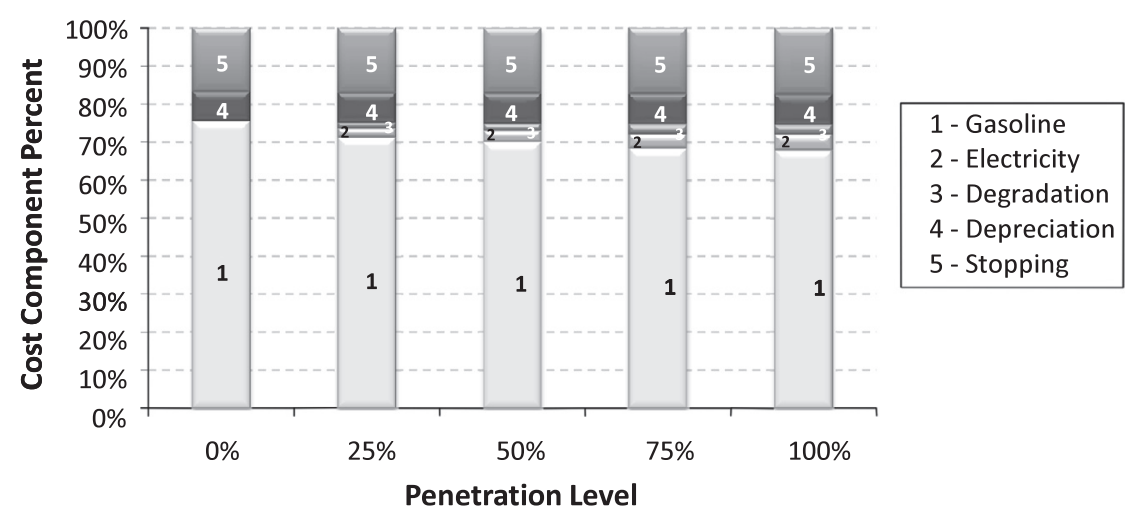

Fig. 8. The effect of charging station penetration level on the cost components for $500 \uparrow$ stopping cost. 
Lastly, we investigate the change of cost components with increasing penetration level. Figs. 7 and 8 depict the percentage of cost components with increasing penetration level for $0 ₫$ and $500 \llbracket$ stopping cost values, respectively. The effect of penetration level is significant for no stopping cost and the gasoline usage significantly diminishes for $100 \%$ penetration level. On the other hand, gasoline is the main source of energy for every penetration level for high stopping costs as depicted in Fig. 8 and the PHEV is mainly driven in CS mode.

In the literature, several studies including Wang and Lin (2009) and Romm (2006) argue that the main barrier for the growth of PHEVs on the road is the scarcity of a charging station in the road network. However, our results show that increasing the penetration level of the charging station infrastructure might not be enough for promoting PHEVs and the tolerance for stopping needs to be taken into account as well. For drivers with less tolerance for stopping, increasing the battery capacity of a PHEV is more important than increasing the number of charging stations. This result might affect each of the stake holders, namely potential PHEV users, infrastructure investors and governments.

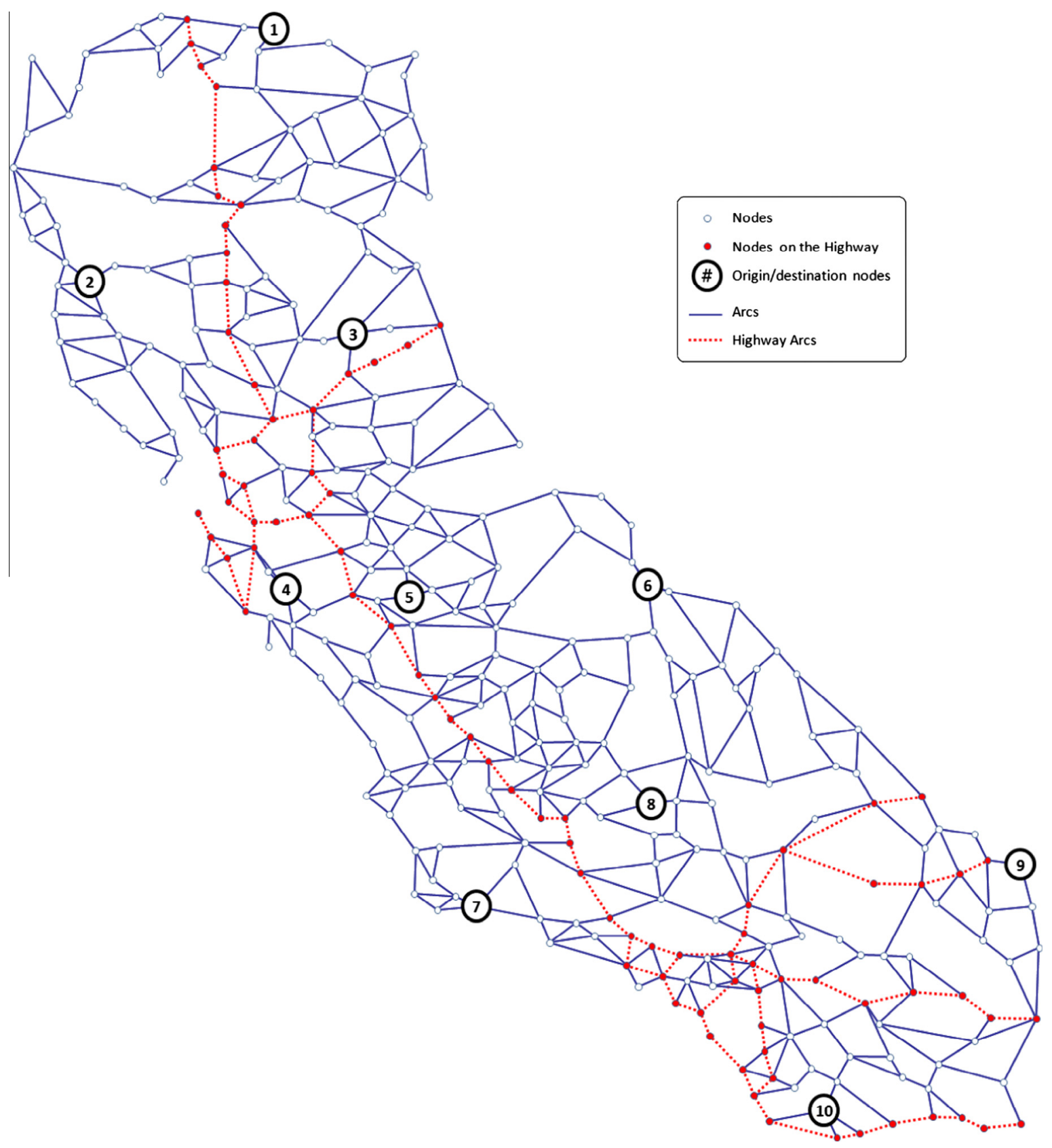

Fig. 9. California network with 339 nodes and 1234 arcs. 
More detailed analyses on the impacts of battery characteristics, driver preferences and road network features on travel costs of a PHEV for long-distance trips is carried out by Arslan et al. (2014) using the presented problem and the solution methodology.

\subsection{California road network}

In order to test the performances of the solution techniques in larger problems and validate the results that we obtained in the randomly generated mesh networks, we consider a real-world California road network (Li and Kollios, 2007). After processing this network, we have 339 nodes and 1234 arcs as depicted in Fig. 9. It is assumed that there is a refueling station located at every node, and the nodes on the highway also have charging stations. The gasoline prices and the electricity prices change uniformly in \$3.5 and \$4.1 range and $10 \leftarrow$ and $12 \leftarrow$ range, respectively. More waiting in the refueling/charging stations might significantly affect the driver tolerance for the stops hence change the stopping cost in our model. In order to take this factor into account and increase the realism of the problem instances we consider varying stopping costs for the California Road Network problem instances. Since larger cities inherently mean more time waiting to get service, we associated stopping costs with population numbers. For the nodes with an urban population of 50,000 or less according to U.S. Census Bureau (2010), the stopping cost is assumed to be 50 c. For those nodes with an urban population more than 50,000 , the cost ranges between $50 ₫$ and $500 ₫$ in proportion with the population numbers. For these settings, we have obtained the minimum cost path and refueling/charging policies for each origin-destination pair between 10 randomly selected nodes as depicted in Fig. 9.

The results of the average of 45 runs are presented in Table 2. For the MIQCP solutions, 27 of the 45 runs resulted in optimal solutions in 60-min time limit. For the remaining 18 runs, the average optimality gap after 60 -min was $19.69 \%$. In the table, all the statistics are given with respect to the MIQCP results. This justifies the negative average gap of DHE4 solution.

Computational performances for the proposed solution techniques were essentially similar to the ones in the previous subsection. It is noteworthy that, from both the solution quality and the time perspectives, DHE1 and SP techniques perform reasonably well in these large problem instances. Not only the computational performances but also practical insights we derive in the mesh network cases remain intact. Similar to Fig. 6, the cost per mile values decrease with increasing CD mode

Table 2

Computational results for California road network.

\begin{tabular}{|c|c|c|c|c|c|c|}
\hline $\begin{array}{l}\text { Solution } \\
\text { technique }\end{array}$ & $\begin{array}{l}\text { MIQCP sol. found } \\
(\%)\end{array}$ & $\begin{array}{l}\text { Average gap } \\
(\%)\end{array}$ & $\begin{array}{l}\text { MIQCP sol. path } \\
\text { found }(\%)\end{array}$ & $\begin{array}{l}\text { Is shortest path? } \\
(\%)\end{array}$ & $\begin{array}{l}\text { Avg deviation from the shortest } \\
\text { path (\%) }\end{array}$ & $\begin{array}{l}\text { Solution time } \\
\text { (s) }\end{array}$ \\
\hline MIQCP & 100.0 & 0.000 & 100.0 & 77.8 & 1.537 & 1684.612 \\
\hline DH4 & 13.3 & 1.672 & 97.8 & 80.0 & 1.517 & 289.142 \\
\hline DH1 & 13.3 & 2.314 & 82.2 & 77.8 & 14.858 & 0.867 \\
\hline DHE4 & 95.6 & -0.010 & 97.8 & 80.0 & 1.517 & 291.179 \\
\hline DHE1 & 82.2 & 0.026 & 82.2 & 77.8 & 14.858 & 2.628 \\
\hline SP & 77.8 & 0.272 & 77.8 & 100.0 & 0.000 & 1.684 \\
\hline
\end{tabular}

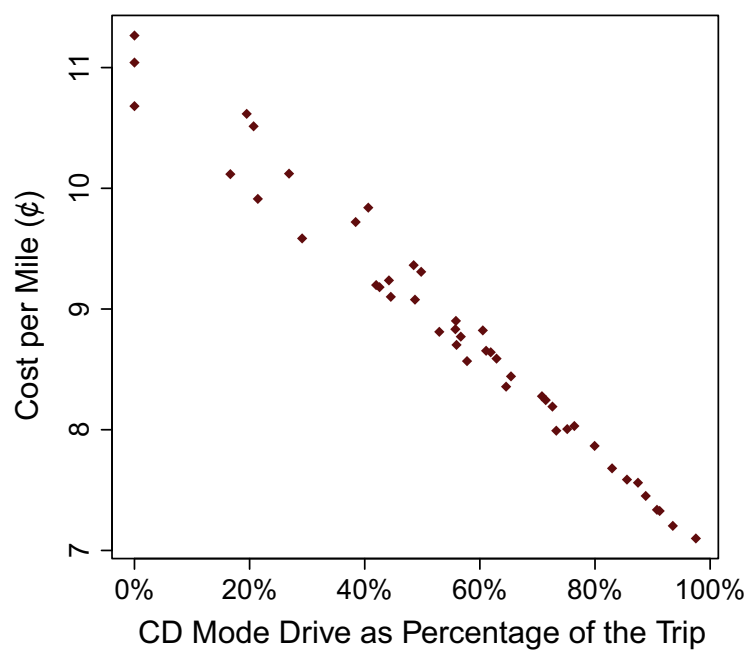

Fig. 10. Cost per mile values for 45 run results in California Dataset. 
travel percentage in Fig. 10. This indicates that the results and insights discussed in this study are quite robust against the changes in the underlying network topology.

Fig. 10 depicts an interesting plot which shows that for every additional 25\% CD mode trip, approximately 1 cent per mile is saved. Even though this might not be a significant decrease for individual drivers, it is of great value from the fleet managers' and governments' perspective taking into account the 575 billion miles long-distance travels in a year (U.S. Department of Transportation, 2010).

\section{Conclusion}

In this article, we introduce the minimum cost path problem for Plug-in Hybrid Electric Vehicles. Urban (i.e. short distance) uses of PHEVs have been extensively investigated with all its expected benefits and shortcomings. However, such a comprehensive scientific treatment does not exist for the long-distance trips. As such, utilization of PHEVs in the long-distance transportation appears as an emerging trend in the logistics. The dual-energy source flexibility, however, begets theoretical challenges to solve the minimum cost path problem for PHEVs. In fact, we show that this problem is $\mathrm{NP}$-complete even though there are polynomial time algorithms to solve its single energy source (electricity or gasoline) special cases. Fluctuations in fuel/electricity costs, battery degradation issues and scarcity of charging stations add further challenges to our problem. In our study, we propose exact and heuristic solution techniques to solve this challenging problem. From a practical point of view, our unifying approach reveals important results about the long-distance use of PHEVs both as personal vehicles and business assets.

We conduct extensive computational experiments both to test the computational performance of the proposed solution methodologies and to drive practical results about the long-distance use of PHEVs. Computational studies show that the proposed MIQCP formulation can solve problems with realistic sizes. A dynamic programming based heuristic and a shortest path heuristic methodologies further extend the sizes of the solvable problems drastically and produce near optimal solutions.

Our results underline a significant advantage of PHEVs that they are quite flexible in their use of gasoline and electricity energy. When the cost of electricity is low compared to the gasoline prices, availability of charging infrastructure and driver tolerance for frequent refueling/charging stops is high, PHEVs increase the share of CD-mode drive which entails lower costs and higher environmental benefits. On the other hand, when these conditions are not met, PHEVs mainly operate in CS mode which enables them to still drive the long-distance trips but limits the realization of the potential cost and emission benefits. The contribution of our study is to present a way to quantify the touted benefits of PHEVs for different scenarios. Therefore, the MCPP-PHEV and the solution methods proposed in this study can be a very useful decision support tool for the personal vehicle owners who want to better understand PHEV economics, for the investors who want to asses the possible benefits of incorporating this new technology in their fleets and for the governments who want to better direct incentives to facilitate the transition from the fossil fuels to more economic, secure and environment friendly energy sources.

The methodologies that we present in this article are not only applicable for PHEVs, but also for all types of hybrid vehicles that run on two types of energy resources. Note that our solution methodologies naturally encompass single energy source alternative fuel vehicles such as electric and hydrogen. Our study reveals one strategic insight about all those alternative energy vehicles: In the literature, most of the studies related to alternative energy vehicles - EV and PHEV in particular - discuss the problem of availability of refueling and charging stations as a barrier to proliferation of those vehicles. However, the limited range of a non-fossil-fuel-energy drive not only brings the problem of finding charging stations on the route, but also results in frequent charging stops which may not be preferable for most of the drivers. Our study shows that this neglected problem can also be a significant barrier. These are significant results for both infrastructure investors and governments. Decision makers need to consider the drivers' tolerance for stopping and more research must be directed towards determining the utility functions of the drivers' willingness for making frequent stops.

\section{Acknowledgments}

This work was supported by the Scientific and Technological Research Council of Turkey (TUBITAK) under the grant number ARDEB-214M211. We are also thankful to anonymous referees for their constructive feedbacks in leading to the current form of this article.

\section{Appendix A. Handling non-simple paths in the mathematical model}

We demonstrate examples of non-simple paths that might appear as the optimal solutions of the E-MCPP-PHEV problem and present methods to handle these non-simple paths by the mathematical model presented in Section 3.1.

First, note that all of the non-simple paths can be handled by duplicating every node in the graph $G$ (as many times as the drivers are willing to revisit the same node in the same trip or as the number of nodes in the worst case). But this implies a much larger graph size and brings along computational burden. Thus, we first present ways to handle those cases by modifying the input graph for the MIQCP model before resorting to the costly node duplication. 


\section{A.1. Problem instance}

- We consider the vehicle instance $V=\langle\bar{P}=1, \underline{P}=0, \bar{G}=9, \underline{G}=0, \varepsilon=1, \rho=1\rangle$. Thus the gasoline range of a PHEV is 9 miles and the electricity range is 1 mile.

- To illustrate different cases, we use a different network instances for each of the three examples (Fig. 11). In all three networks, nodes $A, B$ and $C$ are points on the highway.

- The problem instance is given as $\langle V, X, A, C, 1,9,0,0\rangle$ where $X$ is the input network instance. For the sake of simplicity, we also assume that battery degradation, vehicle depreciation and stopping costs are all zero.

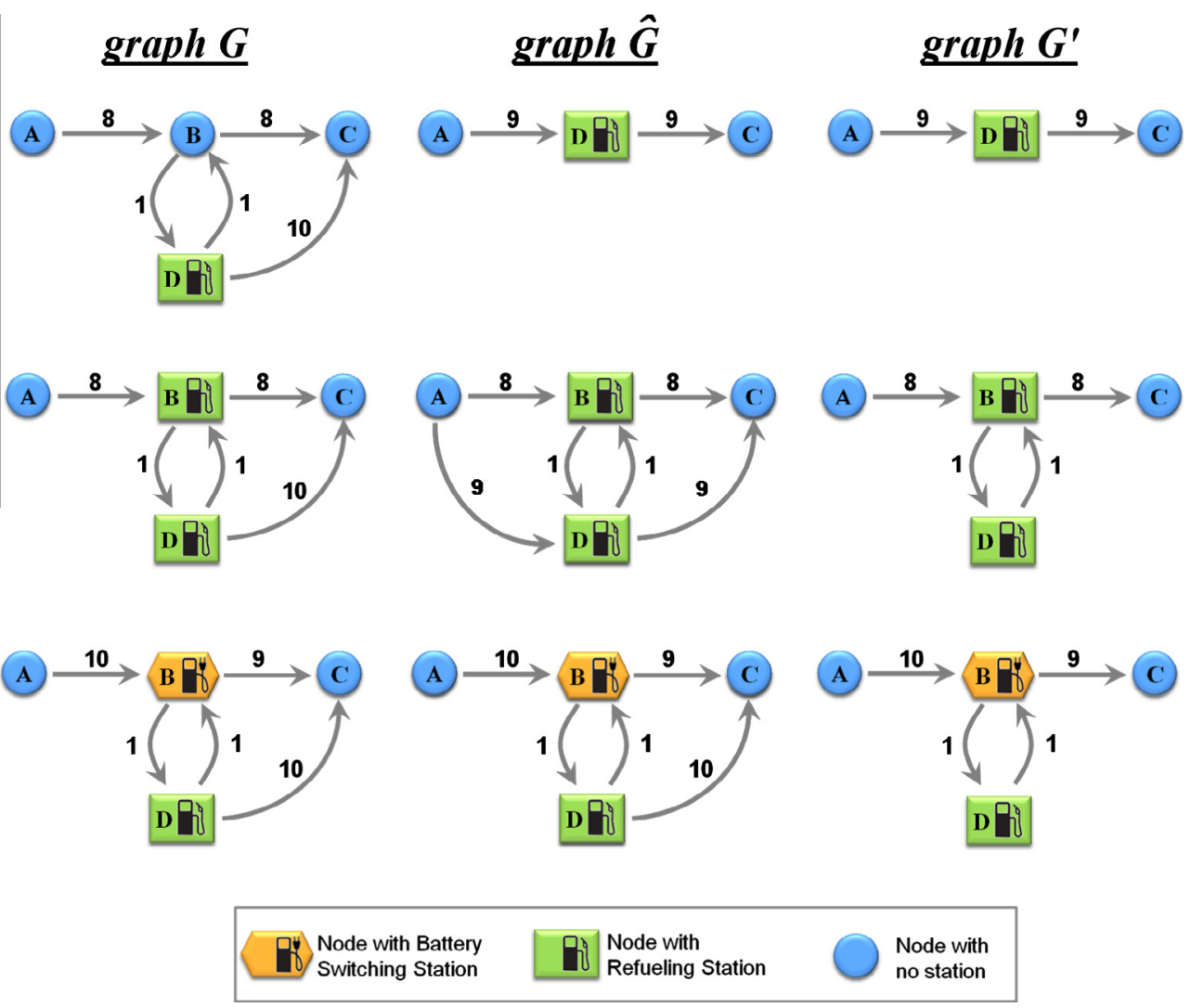

Fig. 11. Non-simple path examples.

Case-1: Detour from the highway to refuel

We can think of nodes $A, B$ and $C$ as points on the highway, and $D$ is a refueling station just one mile away from the highway. Node $B$ is deleted in the meta-network or reduced meta-network since it does not have a station. The optimal non-simple path from $A$ to $C$ in $G$ is $A \rightarrow B \rightarrow D \rightarrow B \rightarrow C$ and can be attained by using the reduced meta-network $G^{\prime}$ as input to the MIQCP formulation.

Case-2: Detour from the highway to refuel in a cheaper station

The middle figure illustrates a detour from the highway. But this time, there is a refueling station on the highway (possibly with a more expensive gasoline price) at which the PHEV can detour and go to node $D$ in order to refuel. In this case, MIQCP formulation can handle the optimal non-simple path $A \rightarrow B \rightarrow D \rightarrow B \rightarrow C$ by using meta-network $\hat{G}$ as the input graph. Indeed, simple path $A \rightarrow D \rightarrow C$ in $\hat{G}$ will correspond to this solution.

Case-3: Refuel twice in the same station

Now, consider the bottom figure. This time, node $B$ has a charging station and node $D$ has a refueling station. Observe that there is only one feasible solution for this problem: $A \rightarrow B \rightarrow D \rightarrow B \rightarrow C$. The PHEV charges battery at node $B$, travels to node $D$ to refuel. Then it necessarily returns back to node $B$ and charges its battery again in order to be able to reach to node $C$. In this example, the optimal path is a non-simple path in all three graph types and thus, MIQCP formulation can only handle such non-simple paths by a node duplication.

Note that this particular instance can be generalized so that more than two visits to the same node, and hence more than one duplication of the node set, is necessary. Note also that this is a rather rare occurrence. The emergence of such 
non-simple paths is not only due to price differences, but also to range limitations as well. In the example, node $B$ is reachable from node $A$, but node $D$ is not. Considering the combined gasoline and electric range of existing PHEVs, this example is not very representative of the real network instances under our scope.

\section{References}

Adler, J., Mirchandani, P.B., Xue, G., Xia, M., 2013. The electric vehicle shortest walk problem. In: 93th TRB Annual Meetings.

Ahuja, R.K., Orlin, J.B., Pallottino, S., Scutellà, M.G., 2002. Minimum time and minimum cost-path problems in street networks with periodic traffic lights. Transport. Sci. 36, 326-336.

Arslan, O., Yıldız, B., Karaşan, O.E., 2014. Impacts of battery characteristics, driver preferences and road network features on travel costs of a plug-in hybrid electric vehicle (phev) for long-distance trips. Energy Policy 74, 168-178.

Artmeier, A., Haselmayr, J., Leucker, M., Sachenbacher, M., 2010. The shortest path problem revisited: optimal routing for electric vehicles. In: KI 2010 Advances in Artificial Intelligence. Springer, pp. 309-316.

Axsen, J., Kurani, K.S., 2010. Anticipating plug-in hybrid vehicle energy impacts in california: constructing consumer-informed recharge profiles. Transp. Res. Part D: Transp. Environ. 15, 212-219.

Axsen, J., Burke, A., Kurani, K.S., 2008. Batteries for plug-in hybrid electric vehicles (phevs): goals and the state of technology circa 2008.

Bektas, T., Laporte, G., 2011. The pollution-routing problem. Transport. Res. Part B: Meth. 45, 1232-1250.

Bellman, R., 1956. Dynamic programming and lagrange multipliers. Proc. Natl. Acad. Sci. USA 42, 767.

Bloomberg, 2015. Via's lutz eyes annual sales of 50,000 plug-in pickups. <http://www.bloomberg.com/news/articles/2015-01-15/vias-lutz-eyes-annualsales-of-50000-plugin-pickups>.

Business Wire, 2014. Greenkraft inc. and efficient drivetrains, inc. jointly receive grant from california energy commission to develop cng-phev class-4 truck. <http://www.businesswire.com/news/home/20141027006268/en/Greenkraft-Efficient-Drivetrains-Jointly-Receive-Grant-California>.

Chen, S., Ljubić, I., Raghavan, S., 2010. The regenerator location problem. Networks 55, 205-220.

Ćirović, G., Pamučar, D., Boanić, D., 2014. Green logistic vehicle routing problem: routing light delivery vehicles in urban areas using a neuro-fuzzy model. Expert Syst. Appl. 41, 4245-4258.

Clean Transport and Technology Limited, 2015. Uk tax incentives for electric vehicles. <http://www.clean-transport.co.uk/electric-vehicle-tax-incentives> (accessed on 10.03.15).

Cui, X., Kim, H.K., Liu, C., Kao, S.C., Bhaduri, B.L., 2012. Simulating the household plug-in hybrid electric vehicle distribution and its electric distribution network impacts. Transp. Res. Part D: Transp. Environ. 17, 548-554.

Dagsvik, J.K., Wennemo, T., Wetterwald, D.G., Aaberge, R., 2002. Potential demand for alternative fuel vehicles. Transport. Res. Part B: Meth. 36, 361-384.

Davis, B.A., Figliozzi, M.A., 2013. A methodology to evaluate the competitiveness of electric delivery trucks. Transport. Res. Part E: Logist. Transport. Rev. 49, $8-23$.

Demir, E., Bektas, T., Laporte, G., 2012. An adaptive large neighborhood search heuristic for the pollution-routing problem. Eur. J. Oper. Res. 223, 346-359.

Demir, E., Bektaş, T., Laporte, G., 2014. The bi-objective pollution-routing problem. Eur. J. Oper. Res. 232, 464-478.

Desaulniers, G., Villeneuve, D., 2000. The shortest path problem with time windows and linear waiting costs. Transport. Sci. $34,312-319$.

Desrochers, M., Soumis, F., 1989. A column generation approach to the urban transit crew scheduling problem. Transport. Sci. 23, 1-13.

Desrosiers, J., Soumis, F., Desrochers, M., 1984. Routing with time windows by column generation. Networks 14, 545-565.

Eisner, J., Funke, S., Storandt, S., 2011. Optimal route planning for electric vehicles in large networks, in: Proceedings of the Twenty-Fifth AAAI conference on Artificial Intelligence.

Electric Power Research Institute, 2005. Batteries for electric drive vehicles status 2005. Technical Report. Electric Power Research Institute.

Erdoğan, S., Miller-Hooks, E., 2012. A green vehicle routing problem. Transport. Res. Part E: Logist. Transport. Rev. 48, 100-114, Select Papers from the 19th International Symposium on Transportation and Traffic Theory.

Felipe, Ángel, Ortuno, M.T., Righini, G., Tirado, G., 2014. A heuristic approach for the green vehicle routing problem with multiple technologies and partial recharges. Transport. Res. Part E: Logist. Transport. Rev. 71, 111-128.

Flath, C., Ilg, J., Gottwalt, S., Schmeck, H., Weinhardt, C., 2013. Improving electric vehicle charging coordination through area pricing. Available at SSRN 2197327

Franceschetti, A., Honhon, D., Woensel, T.V., Bektaș, T., Laporte, G., 2013. The time-dependent pollution-routing problem. Transport. Res. Part B: Meth. 56, $265-293$.

Garey, M.R., Johnson, D.S., 1979. Computers and Intractability, vol. 174. Freeman, New York.

Goeke, D., Schneider, M., 2015. Routing a mixed fleet of electric and conventional vehicles. Eur. J. Oper. Res. doi:http://dx.doi.org/10.1016/j.ejor.2015.01.049.

Golob, T.F., Gould, J., 1998. Projecting use of electric vehicles from household vehicle trials. Transport. Res. Part B: Meth. 32, $441-454$.

He, F., Wu, D., Yin, Y., Guan, Y., 2013. Optimal deployment of public charging stations for plug-in hybrid electric vehicles. Transport. Res. Part B: Meth. 47, $87-101$.

Ichimori, T., Ishii, H., Nishida, T., 1981. Routing a vehicle with the limitation of fuel. J. Oper. Res. Soc. Jpn. 24, $277-281$.

Jiang, N., Xie, C., 2012. Computing and analyzing equilibrium network flows of gasoline and electric vehicles. In: 92nd TRB Annual Meetings.

Jiang, N., Xie, C., Waller, S.T., 2012. Path-constrained traffic assignment. Transport. Res. Rec.: J. Transport. Res. Board 2283, 25-33.

Jiang, N., Xie, C., Duthie, J.C., Waller, S.T., 2013. A network equilibrium analysis on destination, route and parking choices with mixed gasoline and electric vehicular flows. EURO J. Transport. Logist., 1-38

Juan, A.A., Goentzel, J., Bektaş, T., 2014. Routing fleets with multiple driving ranges: is it possible to use greener fleet configurations? Appl. Soft Comput. 21 84-94.

Khuller, S., Malekian, A., Mestre, J., 2007. To fill or not to fill: the gas station problem. In: Algorithms-ESA 2007. Springer, pp. 534-545.

Kobayashi, Y., Kiyama, N., Aoshima, H., Kashiyama, M., 2011. A route search method for electric vehicles in consideration of range and locations of charging stations. In: Intelligent Vehicles Symposium (IV), 2011 IEEE. IEEE, pp. 920-925.

Koç, C., Bektaş, T., Jabali, O., Laporte, G., 2014. The fleet size and mix pollution-routing problem. Transport. Res. Part B: Meth. 70, $239-254$.

Kramer, R., Subramanian, A., Vidal, T., dos AnjosCabral, F., Cabral, L., 2015. A matheuristic approach for the pollution-routing problem. Eur. J. Oper. Res. 243, 523-539.

Kuby, M., Lim, S., 2005. The flow-refueling location problem for alternative-fuel vehicles. Socio-Econ. Plann. Sci. 39, 125-145.

Laporte, G., Pascoal, M., 2011. Minimum cost path problems with relays. Comput. Oper. Res. 38, 165-173.

Li, S., Huang, Y., 2014. Heuristic approaches for the flow-based set covering problem with deviation paths. Transport. Res. Part E: Logist. Transport. Rev. 72. $144-158$.

Li, F., Kollios, G., 2007. Real datasets for spatial databases. <http://www.cs.utah.edu/ lifeifei/SpatialDataset.htm>.

Lin, S.H., 2008a. Finding optimal refueling policies: a dynamic programming approach. J. Comput. Sci. Coll. 23, 272-279.

Lin, S.H., 2008b. Finding optimal refueling policies in transportation networks. In: Algorithmic Aspects in Information and Management. Springer, pp. 280291.

Lin, S.H., 2012. Vehicle refueling planning for point-to-point delivery by motor carriers. In: Proc.2012 IEEE International Conference on Industrial Engineering and Engineering Management, pp. 187-191.

Lin, S.H., Gertsch, N., Russell, J.R., 2007. A linear-time algorithm for finding optimal vehicle refueling policies. Oper. Res. Lett. 35, 290-296.

Lin, C., Choy, K., Ho, G., Chung, S., Lam, H., 2014. Survey of green vehicle routing problem: past and future trends. Expert Syst. Appl. 41, 1118-1138. 
Lopes, M.M., Moura, F., Martinez, L.M., 2014. A rule-based approach for determining the plausible universe of electric vehicle buyers in the lisbon metropolitan area. Transport. Res. Part A: Policy Pract. 59, 22-36.

Mabit, S.L., Fosgerau, M., 2011. Demand for alternative-fuel vehicles when registration taxes are high. Transp. Res. Part D: Transp. Environ. 16, 225-231.

Markel, T., Wipke, K., 2001. Modeling grid-connected hybrid electric vehicles using advisor. In: The Sixteenth Annual Battery Conference on Applications and Advances, 2001. IEEE, pp. 23-29.

Millner, A., 2010. Modeling lithium ion battery degradation in electric vehicles. In: 2010 IEEE Conference on Innovative Technologies for an Efficient and Reliable Electricity Supply (CITRES). IEEE, pp. 349-356.

Mitsubishi Cars, 2015. Outlander PHEV. <http://mitsubishi-cars.co.uk/outlander/explore-phev.aspx> (accessed on 01.03.2015).

Navigant Research, 2013. Selecting electric vehicles for fleets. <http://www.navigantresearch.com/wp-assets/uploads/2013/02/RB-SEVF-13-ExecutiveSummary.pdf>.

Odyne Systems, 2015. Odyne, plug-in hybrid systems for medium- and heavy-duty trucks. <http://www.smithelectric.com/smith-vehicles/>.

Parish, R., Pitkanen, W., 2012. Demand assessment of first-mover hybrid and electric truck fleets. CALSTART. <http://www.calstart.org/Libraries/ Publications/Demand_Assessment_of_First-Mover_Hybrid_and_Electric_Truck_Fleets.sflb.ashx> 8, 2014.

Pelletier, S., Jabali, O., Laporte, G., 2014. Goods distribution with electric vehicles: Review and research perspectives. Technical Report. Technical Report CIRRELT-2014-44, CIRRELT, Montréal, Canada.

Pistoia, G., 2010. Electric and hybrid vehicles: Power sources, models, sustainability, infrastructure and the market. Access Online via Elsevier.

Quantum Fuel Systems, 2012. Quantum announces first delivery of plug-in hybrid electric f-150 pick up truck to florida power \& light. <http://www. qtww.com/press-releases?view=104>.

Romm, J., 2006. The car and fuel of the future. Energy Policy 34, 2609-2614.

Sachenbacher, M., Leucker, M., Artmeier, A., Haselmayr, J., 2011. Efficient energy-optimal routing for electric vehicles, in: Twenty-Fifth AAAI Conference on Artificial Intelligence.

Sathaye, N., 2014. The optimal design and cost implications of electric vehicle taxi systems. Transp. Res. Part B: Meth. 67, $264-283$.

Sathaye, N., Kelley, S., 2013. An approach for the optimal planning of electric vehicle infrastructure for highway corridors. Transp. Res. Part E: Logist. Transp. Rev. 59, 15-33.

Schneider, M., Stenger, A., Goeke, D., 2014. The electric vehicle-routing problem with time windows and recharging stations. Transp. Sci. 48, 500-520. http://dx.doi.org/10.1287/trsc.2013.0490.

Siddiqi, U.F., Shiraishi, Y., Sait, S.M., 2011. Multi-constrained route optimization for electric vehicles (evs) using particle swarm optimization (pso). In: 2011 11 th International Conference on Intelligent Systems Design and Applications (ISDA). IEEE, pp. 391-396.

Sioshansi, R., 2012. Or forummodeling the impacts of electricity tariffs on plug-in hybrid electric vehicle charging, costs, and emissions. Oper. Res. 60, 506516.

Sioshansi, R., Denholm, P., 2010. The value of plug-in hybrid electric vehicles as grid resources. Energy J. $31,1-24$.

Smith Electric Vehicles, 2015. Smith electric vehicles. <http://www.smithelectric.com/smith-vehicles/>.

Smith, O.J., Boland, N., Waterer, H., 2012. Solving shortest path problems with a weight constraint and replenishment arcs. Comput. Oper. Res. 39, 964-984.

Suzuki, Y., 2008. A generic model of motor-carrier fuel optimization. Nav. Res. Logist. (NRL) 55, 737-746.

Suzuki, Y., 2009. A decision support system of dynamic vehicle refueling. Decis. Support Syst. 46, $522-531$.

Suzuki, Y., 2012. A decision support system of vehicle routing and refueling for motor carriers with time-sensitive demands. Decis. Support Syst. 54 (1), $758-767$.

Tate, E., Harpster, M.O., Savagian, P.J., 2008. The electrification of the automobile: from conventional hybrid, to plug-in hybrids, to extended-range electric vehicles. Technical Report. SAE Technical Paper.

Traut, E., Hendrickson, C., Klampfl, E., Liu, Y., Michalek, J.J., 2011. Optimal design and allocation of electrified vehicles and dedicated charging infrastructure for minimum greenhouse gas emissions, in: TRB Annual Meeting.

Turrentine, T.S., Kurani, K.S., 2007. Car buyers and fuel economy? Energy Policy 35, 1213-1223.

United Kingdom Government, 2012. Plug-in van grant. <https://www.gov.uk/government/publications/plug-in-van-grant> (accessed on 10.03.15).

U.S. Census Bureau, 2010. Urban, urbanized area, urban cluster, and rural population, 2010 and 2000: United states. <http://www.census.gov/geo/reference/ ua/urban-rural-2010.html> (accessed on 18.05.14)

U.S. Department of Energy, 2008. Plug-in hybrid electric vehicle charging infrastructure review. <http://avt.inel.gov/pdf/phev/phevInfrastructureReport08. pdf $>$.

U.S. Department of Energy, 2012. Plug-in electric vehicle handbook for fleet managers. <http://www.afdc.energy.gov/pdfs/pev_handbook.pdf>.

U.S. Department of Energy, 2013. Fuel economy of the 2013 chevrolet volt. <http://www.fueleconomy.gov/feg/noframes/32655.shtml>.

U.S. Department of Transportation, 2010. National household travel survey. <http://nhts.ornl.gov/det/Extraction3.aspx> (accessed on 16.02.14)

Volvo, 2015. Volvo fe hybrid. <http://www.volvotrucks.com/trucks/uk-market/en-gb/trucks/volvo-fe-hybrid/Pages/volvo-fe-hybrid.aspx>.

Wang, Y.W., Lin, C.C., 2009. Locating road-vehicle refueling stations. Transp. Res. Part E: Logist. Transp. Rev. 45, 821-829.

Wang, Y.W., Lin, C.C., 2013. Locating multiple types of recharging stations for battery-powered electric vehicle transport. Transp. Res. Part E: Logist. Transp. Rev. 58, 76-87.

Wang, Y.W., Wang, C.R., 2010. Locating passenger vehicle refueling stations. Transp. Res. Part E: Logist. Transp. Rev. 46, 791-801.

Windecker, A., Ruder, A., 2013. Fuel economy, cost, and greenhouse gas results for alternative fuel vehicles in 2011. Transp. Res. Part D: Transp. Environ. 23, 34-40.

Xi, X., Sioshansi, R., Marano, V., 2013. Simulationoptimization model for location of a public electric vehicle charging infrastructure. Transp. Res. Part D: Transp. Environ. 22, 60-69.

Yang, J., Sun, H., 2015. Battery swap station location-routing problem with capacitated electric vehicles. Comput. Oper. Res. 55, 217-232.

Yıldız, B., Karaşan, O.E., 2015. Regenerator location problem and survivable extensions: a hub covering location perspective. Transp. Res. Part B: Meth. 71, 32-55.

Yıldız, B., Arslan, O., Karaşan, O.E., 2015. A branch and price approach for routing and refueling station location model. Eur. J. Oper. Res. http://dx.doi.org/ 10.1016/j.ejor.2015.05.021. 